Portland State University

PDXScholar

6-27-1986

\title{
Comparative Self-Monitoring Behavior and Recall of Verbal and Nonverbal Interactional Information about Partner in Conversations with Ablebodied and Disabled Partners
}

Doris L. Werkman

Portland State University

Follow this and additional works at: https://pdxscholar.library.pdx.edu/open_access_etds

Part of the Interpersonal and Small Group Communication Commons Let us know how access to this document benefits you.

\section{Recommended Citation}

Werkman, Doris L., "Comparative Self-Monitoring Behavior and Recall of Verbal and Nonverbal Interactional Information about Partner in Conversations with Ablebodied and Disabled Partners" (1986). Dissertations and Theses. Paper 3682.

https://doi.org/10.15760/etd.5566

This Thesis is brought to you for free and open access. It has been accepted for inclusion in Dissertations and Theses by an authorized administrator of PDXScholar. Please contact us if we can make this document more accessible: pdxscholar@pdx.edu. 
ABSTRACT OF THE THESIS OF DOris $\mathrm{L}$. Werkman for the Master of Science in Speech Communication presented June 27] 986$.

Title: Comparative Self-Monitoring Behavior and Recall of Verbal and Nonverbal Interactional Information About Partner in Conversations with Ablebodied and Disabled Partners.

APPROVED BY MEMBERS OF THE THESIS COMMITTEE:

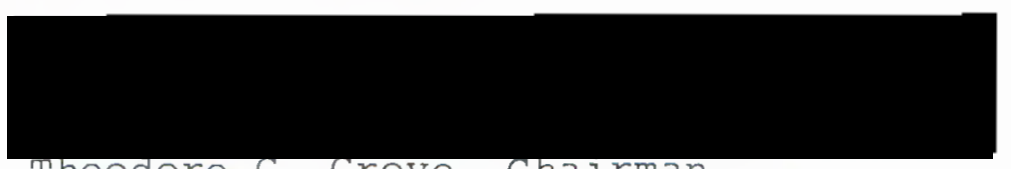

Theodore G. Grove, chairman

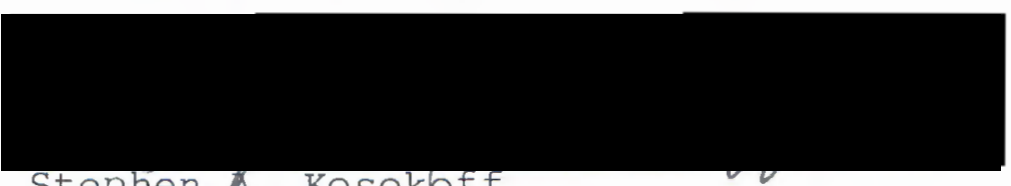

Stephen A. Kosokbit
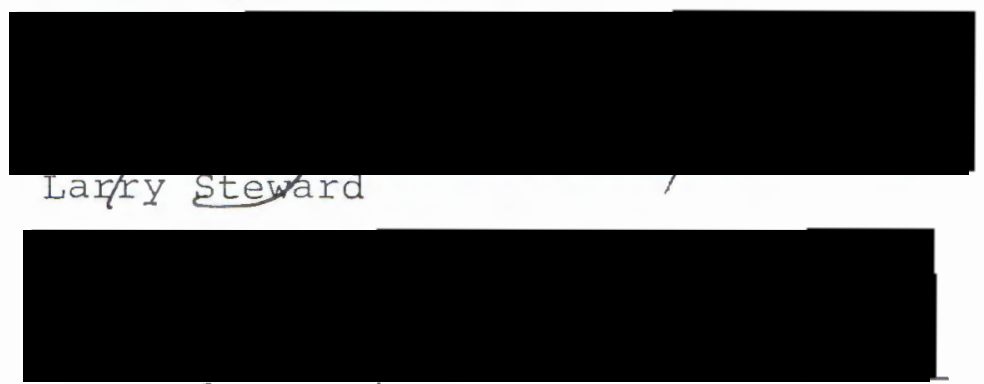

Leonard D. Cain

When stranyers rneet, there is a high level of uncertainty due to the infinite nurber of possible alternatives in behavior between the two people. Previous research 
indicates that communicators will attempt to reduce the level of uncertainty by using available verbal and nonverbal information of the other, by seeking similarities with the other, and by observing the situation itself. This information is used to predict attitudes and beliefs, as well as to attribute characteristics of the other. Other studies indicate that a person will increase her level of self-monitoring activity when the level of uncertainty is increased.

All previous research on uncertainty reduction, attributional processes, and self-monitoring activity has been conducted with regard to the ablebodied population. Comparisons between transactions of ablebodied participants with transactions involving a disabled participant report important differences in communicators' perceptions and behaviors. These differences include an increased level of anxiety, or uncertainty, felt by the ablebodied person. The indication is that ablebodied persons have less success in reducing their level of uncertainty in an interaction with a disabled person. At present, there has been no report of an empirical test of the connections among uncertainty reduction, attributional processes, and self-monitoring activity during transactions with a disabled partner. This research was a rudimentary effort at model-building in this area, along with three exploratory probes of a heuristic model. 
In order to assess the effects of interacting with a disabled partner on self-monitoring activity, on awareness of partner behavior, and to assess the relationship between self-monitoring and recall of partner information, the investigator conducted an experiment whereby videotaped conversations with ablebodied partners and with disabled partners were compared. subjects completed three recall-of-information questionnaires pertaining to their own and their partners' communication behavior. Questionnaire responses were scored by comparing recalled behavior with content analysis of videotapes of the same conversations. The actual experiment consisted of 1) recruitment and videotaping of subjects, 2) training of confederates, 3) development of three questionnaires, 4) training of coders, 5) assessment of interrater reliability, and 6) administration and subsequent analyses of data.

Verbal and nonverbal scoring categories were used to detail the level of recall of partners' verbal, partners' nonverbal, and subjects' verbal and nonverbal behavior. Three coders were trained to discern verbal and nonverbal behaviors, and to code pairs of five minute conversations each subject had with an ablebodied and a disabled communicator. The derived data were compared with three questionnnaires in order to measure self-monitoring activity and subjects' recall of partners' behavior. 
Results from a correlation analysis indicated a low to moderate positive correlation between the amount of selfmonitoring activity and levels of information during the interaction with the disabled partner. Self-monitoring activity during the interaction with the disabled was somewhat lower than during the interaction with an ablebodied partner. There was significantly less information recall of the disabled partner than of the ablebodied partner. The empirical evidence generated by this research shows that there is a significant diminution of information about partner when that partner is disabled. 
COMPARATIVE SELF-MONITORING BEHAVIOR AND RECALL OF VERBAL AND NONVERBAL INTERACTIONAL INFORMATION ABOUT PARTNER IN CONVERSATIONS WITH ABLEBODIED AND DISABLED PARTNERS

by

DORIS L. WERKMAN

A thesis submitted in partial fulfillment of the requirements for the degree of

MASTER OF SCIENCE

in

SPEECH COMMUNICATION

Portland State University 
TO THE OFFICE OF GRADUATE STUDIES AND RESEARCH:

The nembers of the committee approve the thesis of Doris L. Werkman presented June 27, ] 986 .

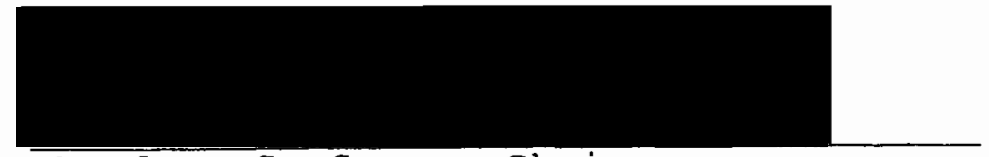

Theodore G. Grove, Chairman

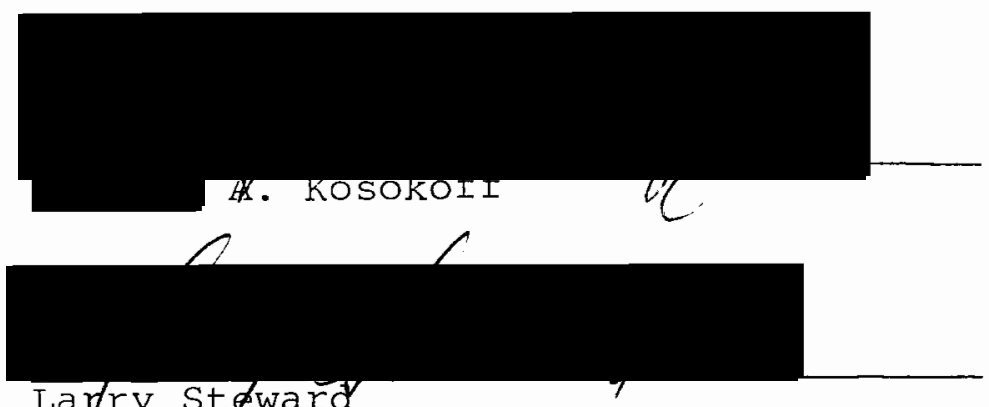

Larlry støward
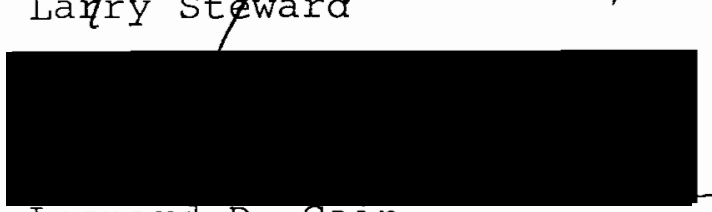

Leonard D. Caın

APPROVED :

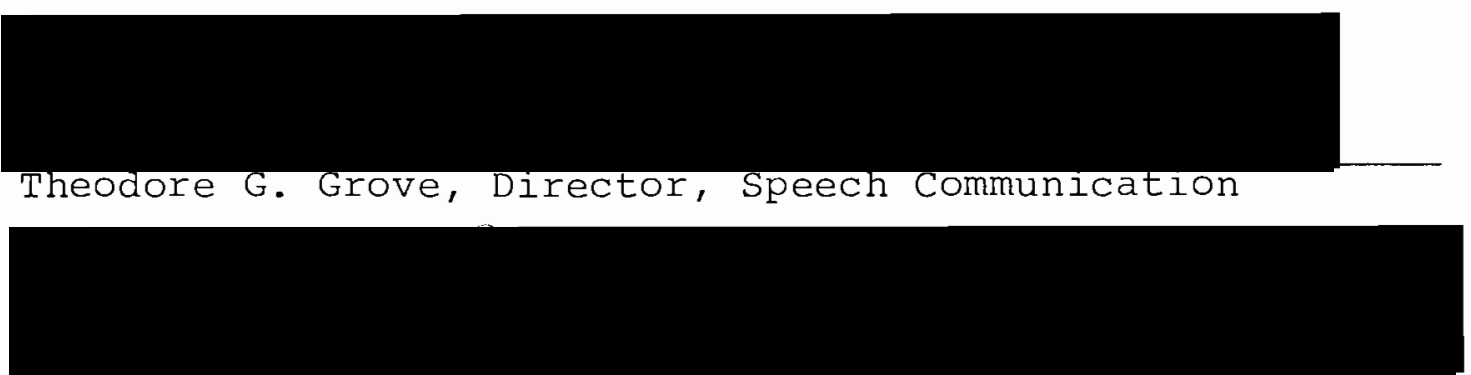

Bernard Ross, Acting Dean, Graduate Studies and Research 


\section{ACKNOWLEDGMENTS}

My sincere appreciation and acknowledgment is extended to all those individuals who were indispensable in the execution of this study.

Special thanks is extended to my thesis committee. Primarily, to Dr. Theodore Grove, my committee chairman and graduate advisor for his empirical expertise, for his emphasis on "just writing" (over and over and over again), and his continual soothing noises when I began to panic. Dr. Stephen Kosokoff is thanked for his constantly opened door and tossed-in humor to help prioritize my life. Dr. Larry Steward has a special thanks for his enthusiasm of my area of interest from the first day I became a graduate student, and also for Dr. Leonard Cain, who added depth to this project with his sociological perspective.

I am amazed at, and forever grateful to, my confederates who took time from their busy schedules to help collect data. A thank you is extended to Julie Vawser, Kathy Brown, Elise Brickman-Schultz, Deborah Turner, and Beth Sager, with a special thanks to confederates Lora sager and Gisele Tierney, who had the misfortune (for them) and the fortune (for me) to have an office located across from mine while data was being collected. I would also like to thank my coders, Sara Miller, Colleen McMoy, and Missy Butler, 
for watching seemingly endless hours of videotaped conversations.

I am overwhelmed by the number of people who have aided me in this effort. My thanks goes to Deborah Swan, Bill and Candy Field, and my parents, Dick and Bette Werkman, as well as the many people in the Department of Speech Communication, who all offered me constant support; and I must not forget Ray Coker, whose thesis became my roommate and model. I also appreciate Pat Hamilton, departmental secretary. Not only was her advice on policy matters invaluable, but her lunches sustained me for two years.

A special acknowledgment goes to my children, Chris and David, who periodically would voice an awed "wow" when needed, and who seldom complained when we had burritos for dinner again. Lastly, I would like to recognize all of my friends who voiced that tiny doubt, "It's not that we don't think you'll finish, but ..." To them I now can confidently say, "...but I did; I finally made it." 
TABLE OF CONTENTS

PAGE

ACKNOWLEDGMENTS.......................

LIST OF TABLES

viii

LIST OF FIGURES...................... ix

CHAPTER

I INTRODUCTION..................... 1

statement of Purpose................. 4

II THEORETICAL FRAMEWORK: RESEARCH IN

REDUCTION OF INTERACTIONAL STRESS.........

Uncertainty reduction............ 7

Attribution processes............. 9

self-monitoring activity........... 12

III THEORETICAL FRAMEWORK : RESEARCH ON

TRANSACTIONS BETWEEN ABLEBODIED AND

DISABLED COMMUNICATORS ..............

Implications of Previous Research:

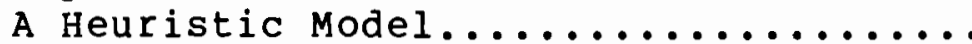

IV METHODS....................... 30

Definition of Theoretical Variables.... 31

Procedural overview.............. 31

Recruitment and Videotaping of

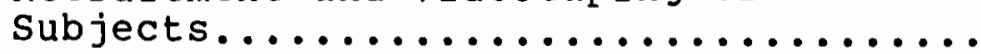

Training of Confederates.......... 36

Instrumentation.............. 36

Development of questionnaires

Training of coders and content

analysis of videotapes 
Transferring coder data to data forms

scoring questionnaire data

Interrater Reliability............

Coder agreement on raw data

Coder agreement on category data

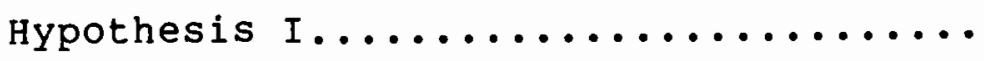

Questionnaire totals

Matched pair analysis

Nonverbal behavior recall

Follow-up data analysis on TABs

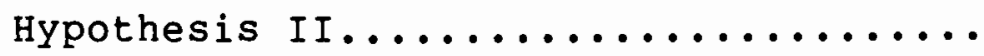

Questionnaire totals

Matched pair analysis

Nonverbal behavior recall

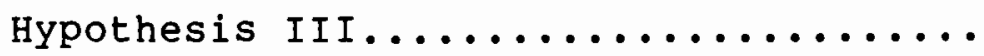

Questionnaire totals for PANB

Questionnaire for PVVB

Matched pair analysis

Nonverbal behavior recall

summary ....................

vi DISCUSSION.................

Credibility of Experiment

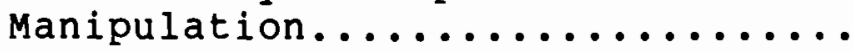

Limitations of the study.........

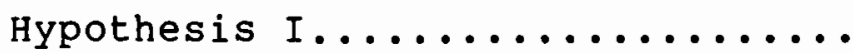

Hypothesis II...............

Hypothesis III..............

General Discussion and Suggestions for Future Research............. 
LIST OF TABLES

TABLE

PAGE

I Pearson Correlation on Ten Matched Pair

Items Associated with self-Monitoring

and Information Recall in Interactions

with Disabled Persons...............

II Follow-Up Correlation on Ten Matched Pair

Items Associated with TABs in

Interactions with Ablebodied Partners....

II T-Tests on SB Totals Associated with TAB

and TAD Treatment conditions...........

IV T-Test on Ten SB Items Associated with

$\mathrm{TAB}$ and TAD Treatment Conditions........

$V$ T-Test on PANB and PVVB Totals Associated

with TAB and TAD Treatment conditions....

VI T-Tests on PANB and PVVB Items Associated

with TAB and TAD Treatment Conditions.... 


\section{LIST OF FIGURES}

FIGURE

PAGE

1

A Heuristic Model...................

25

2

Relationship of self-Monitoring and

Attention to Partner's Behavior.......... 
CHAPTER I

\section{INTRODUCTION}

Humans have a need to make sense of their world (Heider, 1958; Jones, 1971; Kelley, 1971; Kanouse, 1971; Berger, 1975; Berger \& Calabrese, 1975). The level of anxiety felt in any given situation is increased due to the infinite number of choices of behavior, responses, classifications of events that could be used to maintain a sense of equilibrium in one's world. One means of reducing the uncertainty is to attribute meaning to a situation by comparing it to past experiences (Berger \& Calabrese, 1975). When a situation can be fit into an established category, less ambiguity is felt. This lowers the level of anxiety. When beginning an encounter with a stranger, the alternatives for behaving are limitless (Berger \& Calabrese, 1975; Berger, 1975; Heider, 1958). Some theorists believe the early acquaintance phases of an encounter are used to reduce the associated uncertainty during this period. One process used to reduce this uncertainty of behavioral expectations is to predict behaviors and atiitudes of others based on verbal and nonverbal cues. These cues give each participant information by which inferences are drawn about the other. 
Berger and Calabrese (1975) believe the first few minutes of an encounter are used specifically for each communicator to obtain background information about the other. As information is obtained, each participant compares it to her own background. If a greater amount of homophily is perceived, the participants will believe they share attitudes and beliefs (Berger, 1975; Berger \& Calabrese, 1975; Novack \& Lerner, 1968; Byrde, 1961). This belief in hornophily reduces the alternatives of each person's behaviors, thus reducing the uncertainty of relational behavior.

Another means of reducing the anxiety in an encounter is to interpret the causes of behavior of the other (Heider, 1958; Jones, 1971; Kelley, 1971; Kanouse, 1971). By predicting and interpreting the causes of behavior, it is believed one may avoid the embarrassment of failing to meet relational expectations and, therefore, may gain rewards of approval (Kelley, 1971). People desire to manifest their intelligence by showing an understanding of behavioral requirements. By reducing the reasons for behavior, the number of alternatives of possible behaviors are reduced. This will reduce the anxiety and create more comfort during the interaction.

The vast majority of studies reported on both the theory of uncertainty reduction and the theory of attribution have been conducted with ablebodied subjects. One in eleven persons in the United States is labeled as disabled 
(President's Committee on Employment of the Handicapped, 1975). Previous studies comparing transactions between able-bodied individuals to transactions involving one disabled partner report important differences in communicators' perceptions and behaviors (Davis, 1961; Goffman, 1963; Kleck, 1966; Kleck, Ono \& Hastorf, 1966; Kleck, 1975). For example, there is a greater level of anxiety for the ablebodied participant when interacting with a person who is disabled as compared to encounters with an ablebodied peer (Kleck, 1966; Kleck, Ono \& Hastorf, 1966). When there is a choice, ablebodied individuals tend to avoid interacting with someone who is disabled. When the interaction are unavoidable, the ablebodied person will tend to terminate the interaction sooner than during encounters with an ablebodied person. Gestural behaviors and eye contact is exhibited less by the ablebodied participant, and ablebodied communicators will stand at a greater distance from the disabled person.

From these and other findings concerning behavioral and perceptual differences in interactions between ablebodied and disabled individuals from those found in ablebodied dyads, it seems reasonable to ask if the research on uncertainty reduction and attribution processes can be generalized to interactions between ablebodied and disabled communicators. For example, are the verbal and nonverbal cues processed differently by the ablebodied participant? 
Specifically, is the able-bodied person's view of the disabled other's attitudes and belief system influenced by the awareness of the disability? Would the higher level of anxiety felt by the ablebodied person hinder the reception of verbal and nonverbal information normally used by her to reduce uncertainty? Is there a reduction in efficiency of information processing normally employed by ablebodied communicators during the encounter? Do distinctive effects of the presence of the disability impair the uncertainty reduction process that is normally employed to create a smooth and satisfactory interaction for both participants?

\section{Statement of Purpose}

The purpose of this study is to assess some predicted effects of interacting with a physically disabled individual--effects on self-monitoring activity and on information acquisition on the part of the ablebodied communicator. Several strategies used to reduce uncertainty may result in inattention to interactional information. This could result in less reduction of uncertainty and consequent higher uncertainty and, concomitantly, lowered interactional satisfaction. For example, one strategy would include an increased self-monitoring activity on the part of the ablebodied participant. Also, increased attention to (distraction) elements on the visible disability may occur. Both hyper-self-monitoring and distractive 
effects could result in loss of information normally used to reduce uncertainty and coordinate conversation.

The design of this study will require videotaped interactions of ablebodied subjects conversing separately with an ablebodied and a physically disabled stranger. Content analysis of videotapes and examination of post-session questionnaires will be assessed to check selected relationships predicted in an heuristic model and corresponding to the following hypotheses:

Hl: In interactions with a disabled person, there will be a negative correlation between the amount of self-monitoring activity by the ablebodied person and her level of interactional and personal information processing.

H2: Ablebodied persons will engage in more selfmonitoring activity during an interaction with a disabled person than during an interaction with an ablebodied person.

H3: Ablebodied persons will attend to informational sources of uncertainty reduction less during interactions with a disabled person than during an interaction with an ablebodied person.

Essentially, the object of this investigation is to check for predicted differences in the kind of information ablebodied communicators obtain in conversations with disabled partners. Evidence from past research suggests that 
habitual stress-reducing attributions that are successful in most first encounters with strangers tend to increase anxiety in ablebodied/disabled interactions. While several explanations have been suggested through which that outcome occurs, there have been no reports of an empirical test in this area. 
CHAPTER II

THEORETICAL FRAMEWORK:

RESEARCH IN REDUCTION OF INTERACTIONAL STRESS

Uncertainty Reduction

As strangers meet, they typically will have a high level of anxiety due to the infinite number of possible alternatives for their behavior (Heider, 1958; Berger, 1975; Berger \& Calabrese, 1975). They will try to lower the uncertainty of interactional behaviors in order to reduce the level of potential stress. One means of reducing the uncertainty is to find a common ground, or similarities, in attitudes and beliefs (Berger 1975). This perceived hemophilia enables one to engage in predicting which attitudes the other holds. By predicting possible attitudes, people believe they are able to narrow the range of alternatives of the other's future behavior. This aids in the selection of the most appropriate action in order to behave with some degree of confidence which will, in turn, lower the anxiety of unknown relational expectations. Prior studies have revealed the first few minutes of an interaction are used to gain biological and demographic information (Berger, 1975; Berger \& Calabrese, 1975; Berger \& Lamar, 1976). This information is used in the process of reducing uncertainty by comparing one's own background to 
the other's. Perception of similar backgrounds will generate predictions of similar attitudes. When homophily is perceived as high, people believe the other will behave similarly to themselves (Berger \& Calabrese, 1975). When it is perceived as low, it is generally predicted the other holds dissimilar attitudes. This reduces the number of possible alternatives of perceived appropriate responses between the two people.

It has been found that when communicators perceive high homophily, they feel the communication event is positive and this feeling leads to a greater amount of attraction (Irovack \& Lerner, 1968; Byrde, 1961). In turn, this leads to the desire to create more similarity (Novack \& Lemer, 1968). One believes the other is more intelligent, better informed, and more well adjusted than those who are not perceived as homophilious (Byrde, 1961). This also creates a reduction of uncertainty for future behavior (Berger \& Calabrese, 1975). When uncertainty is reduced, there is a greater total amount of verbal and nonverbal expression. This produces more information about the other, increasing the potential for the prediction of future behavior. In relationships where uncertainty has increased after believing one knew the other, the majority of the relationships became less intimate or were terminated (Planalp \& Honeycutt, $1984)$. 
Information that could generate possible predictions of attitudes and behaviors are obtained through both verbal and nonverbal channels and from the situation itself (Berger, 1975). For example, while attending a youth basketball game as an observing parent, a woman in the audience is noticed. It may be assumed she is a parent like yourself. Watching the woman cheer and call plays, it could be inferred she enjoys sports and is a supportive parent. Her apparent enthusiasm leads you to think her attitude toward sports is positive. She could be a health advocate who actively supports youth sporting events.

Work on uncertainty reduction has found that such contextual information allows individuals to form inferences before the interaction actually begins (Berger, 1975). These inferences are believed to aid communicators in their understanding of the encounter, thus enabling them to behave with some degree of confidence that their behavior will engender predictably-appropriate responses from the other. The totality of this process is to lead toward a smooth and satisfactory interaction. For example, "children," "sports," and "health clubs" may be appropriate topics of discussion with the woman at the basketball game.

Attributable Processes

The process of attribution is another mechanism which communicators use to reduce uncertainty. Traditionally, 
the theory of attribution deals with the rules an average person uses in an attempt to interpret the causes of observed behavior (Heider, 1958; Jones, 1971; Kelley, 1971; Kanouse, 1971). It is based, in part, on the idea that people will predict attitudes and behaviors of others based on both verbal and non-verbal cues in an encounter. This aids in creating a balance between what one believes to be true of the world and what one observes in the world (Heider, 1958). People attribute characteristics, intentions, feelings, and traits to others in an attempt to make sense of the world (Kanouse, 1971). The content of the situation must be in balance with the content to maintain an equilibrium with one's basic understanding of the world (Heider, 1958). Heider believes people have a definite idea of how the world fits together -- it's not arbitrarily put together. The features of this are in some way internalized, creating a cognitive matrix that underlies one's interpretation of another's behavior. This is used to generate predictions of another's future behavior (Kelley, 1971). With these predictions, strangers are able to lower the anxiety felt in an interaction by reducing some of the ambiguity of behavioral expectations.

It is important for people to avoid the embarrassment of failing to meet unspoken relational expectations by showing an understanding of the requirements (Kelley, 1971). People also want to gain rewards of approval. By reducing 
the number of possible alternatives in behaviors, these goals are perceived as more attainable. Where there is the potential or several adequate inferences based on available information, people will generally use the cues most heavily influenced by the situation (Kanouse, 1971; Goffman, 1967). Once the conclusion is formed, people are unlikely to give other alternatives much consideration.

Information used to interpret the causes of events is gained through both verbal and nonverbal channels (Heider, 1958; Kelley, 1971; Kanouse, 1971). For example, the woman seen at the youth basketball game could be explained by inferring she is an observing parent because that's why you are there. As she cheers and calls out plays, it could be concluded she is involved with her children and possibly with other community organizations such as the P.T.A.

With such contextual information, people will then behave in what they perceive to be the correct manner until new information enters their awareness (Goffman, 1967; Kanouse, 1971). These predictions are based on the perceived adequacy of information gathered by the participants (Kelley, 1971).

For the individual, a reduction of uncertainty and increased attributional confidence are the same in that people believe they are able to predict the other's behaviors and attitudes (Clatterbuck, 1979; Berger \& Calabrese, 1975). They believe this imperfect knowledge of the other 
enables them to guide their own behavior during the encounter (Kelley, 1971; Berger \& Calabrese, 1975). As new information about the other is considered, and it contradicts previous perceptions, the uncertainty of the encounter is increased. In order to reduce this anxiety, this information will either be incorporated into the perception of the other, to it will be rejected in order to maintain the balance between the context and the content (Heider, 1958).

\section{Self-Monitoring Activity}

Many theorists believe people constantly evaluate, reevaluate, and draw conclusions of perceived traits of others to create a smooth and satisfactory interaction (Heider, 1958; Jones, 1971; Kelley, 1971; Kanouse, 1971; Berger, 1975; Berger \& Calabrese, 1975). It is found that much of this process is out of their awareness (Langer, 1978; Berger \& Douglas, 1982;). People often engage in "mindless" interactions that require low levels of attention and awareness. There is often the use of standardized, recurring scripts and habitual behaviors.

An awareness of the process of evaluations and the preplanning of scripts produces an inordinate amount of selfmonitoring behavior (Berger \& Douglas, 1982). This happens when (1) it is a novel situation and there is no appropriate script available; (2) external factors prevent the completion of a script; (3) scripted behavior becomes 
effortful due to more behavioral requirements; (4) a discrepant outcome is expected; and (5) there are multiple scripts that come into conflict and none of them can be enacted.

A "high" self-monitoring communicator tends to be conscious of others, adaptable to the situation and to the other, and sensitive to other's communication needs (snyder, 1974). Within these limits, self-monitoring allows the communicator to effectively adapt to diverse communication situations, improve listening ability, and maintain awareness of the other. However, Spitzberg and Cupach (1984, p. 82) believe it "may be naive to assume that consistent high awareness is the most competent state of affairs; consistent high awareness might paradoxically lead to inefficient communication under certain circumstances."

Taken to an extreme, self-monitoring activity can cause a dysfunction in communication (Lofland, 1976). The communicator can become extremely self-aware and obsessed with analyzing every detail of an interaction to the point of distraction and incompetence. This could result in a lack of spontaneity in interactional involvement. But, most important to the present study is that hyper-self-monitoring activity could result in one's inattention to information necessary for the well-coordinated interaction -- personal information about the other as well as information about how the other interacts. 
The distinction between a communicator labeled as a "high" self-monitor and one who engages in hyper-self-monitoring activity, or too much activity, is that the "high" monitor focuses on the other and the interaction. The communicator who is overly active in self-monitoring focuses almost exclusively on the self. In small degrees, effective adaptation to others is promoted; large degrees could result in a preoccupation with the self. In turn, this could interfere with good listening for a consequent loss of information about the other that personalizes the interaction.

There are several forms of alienation that can alter the focus of the interaction and distract a communicator (Goffman, 1967; Lange, 1973). For example, people who enter an interaction highly embarrassed or anxious tend to be overly self-conscious. Also, people who are overly "other conscious," focusing on specific traits of the other such as perceived insincerity or immodesty, will become more aware of their own behavior. Goffman (1967) states that such contextual stimuli can alter the focus of the interaction. This causes an increase of self-monitoring activity. For these reasons, extremes of self-monitoring behavior in the interaction can create a corresponding increase in anxiety. 
THEORETICAL FRAMEWORK: RESEARCH ON TRANSACTIONS BETWEEN ABLEBODIED AND DISABLED COMMUNICATORS

A person with a visible, physical disability is often stigmatized or highly discredited by society (Davis, 1961; Goffman, 1963; Kleck, 1966; Kleck, Ono \&.Hastorf, 1966; Gleidman, 1979; Dahnke, 1983. This minority, which consists of an estimated 20 million people in the United states, is often discriminated against -- handicapped by society due to their disability (Davis, 1961; Goffman, 1963; Gleidman, 1979; President's Committee on the Employment of the Handicapped, 1975;). The areas of discrimination are wide, including education, economics, socialization, and employment.

Prejudice toward disabled persons is deeply entrenched in the American culture where there is a strong, rigid standard of personal integrity and physical soundness (Goffman, 1963; Van Riper \& Emerson, 1984). Deviance from these standards is regarded negatively by society through social stigmatization.

Historically, a person with any type of disability was rejected by society. For survival purposes, only the most able of humans were allowed to eat what was hunted and gathered. At the time of the old Testament, it was 
believed a disability represented divine punishment for sins committed (Van Riper \& Emerson, 1984).

During the Middle Ages, most disabled persons were thought to be possessed by evil spirits. They were confined to their homes and publicly stoned if they ventured into the public eye. The alternative to this confinement was to become a source of humor - the court jester or clown (Van Riper \& Emerson, 1984).

As late as twenty years ago, many disabled people were imprisoned, kept in mental institutions, or placed in nursing homes because society was unsure of what to do with them. Van Riper \& Emerson (1984) believe the contemporary feelings of pity toward disabled persons are due to organized religion which constantly preaches the need of compassion toward those thought of as "less fortunate."

Strong societal pressures for independence as perceived by a disabled person can contribute to feelings of lowered self-esteem (Davis, 1961; Goffman, 1963; Dahnke, 1983. Negative aspects of a lowered self-esteem are reinforced by general attitudinal factors of ablebodied persons. Low self-esteem tends to impede normal social interactions (Goffman, 1963). As a result, disabled individuals have virtually no marketing identity and are considered a nonentity in the business world (Ruffner, 1982). These factors may restrict the person from reaching her full potential as a human being and prevent her from fully 
entering into the mainstream of society (Goffman, 1963; Dahnke, 1983).

Previous studies of transactions between ablebodied and disabled communicators (TADS) as compared to transactions between ablebodied peers (TABs) indicate an extensive array of aberrations in interactional behaviors and perceptions of the communicators (Davis, 1961; Goffman, 1963; Kleck, 1966; Kleck, Ono \& Hastorf, 1966; Thompson, 1972; Kleck, 1975; Dahnke, 1983). It has been found there is a greater emotional arousal during TADs on the part of the ablebodied person than during TABS (Kleck, 1966). Using a Psychoglavanic skin Response measurement, kleck found ablebodied individuals registered a lower resistance while interacting with a disabled person, indicating a greater emotional response. Goffman (1963) calls this the pathology of interaction. There is some evidence that extremely high autonomic arousal in initial encounters is associated with discomfort and a preference for avoiding future contact with the person perceived to have caused the discomfort (Lange \& Grove, 1981).

When there is a choice in interactions, ablebodied communicators tend to avoid interacting with someone disabled (Davis, 1961; Goffman, 1963; Kleck,1966). Then TADs cannot be avoided, the ablebodied participant tends to terminate the interaction sooner than in TABs (Goffman, 1963; Kleck, 1966; Kleck, Ono \& Hastrof, 1966). During interactions, 
the physical distance between the two communicators will increase (Kleck, 1966; Kleck, Ono \& Hastrof, 1966). The ablebodied person will decrease eye contact. exhibit less gestural behavior, and decrease her range of vocal variation (Kleck, 1966; Kleck, Ono \& Hastorf, 1966; Kleck, 1975). Ablebodied participants also tend to alter their beliefs to some degree. For example, they will say that sports and dancing are not important when, in fact, they are important (Kleck, Ono \& Hastorf, 1966).

In addition, there is a great deal more interactional stress and tension during face-to-face encounters (Davis, 1961; Goffman, 1963, Kleck, 1966; Gleidman, 1979). When unease is experienced during TADs, the ablebodied participant tends to place the burden of reducing the stress on the disabled person (Davis, 1961; Goffman, 1963). Perhaps ablebodied persons feel the disabled partner has had more experience in lowering interactional stress caused by the distracting effect of the disability.

The stress in TADs may also be a result of the uncertainty of the most appropriate communication strategy to use for both participants (Dahnke, 1983). The disabled person is often uncertain in how she is perceived and received by the able-bodied communicator (Davis, 1961; Goffman, 1963). If someone is nice toward her, she may wonder what is underlying the gesture. There seems to be no ready-made shortcut for anticipating quality and degree of acceptance. 
For example, "a Southerner can't treat a Black person equally," or "working class people think intellectuals are weaklings" might be considered a shortcut to others' perception.

The ablebodied person is also uncertain about what is appropriate behavior. There is a general norm in this culture that dictates that people behave with kindness toward others, especially toward people considered "less fortunate" (Davis, 1961). On one hand, the ablebodied person cannot allow herself to make direct sympathetic responses as she may be overstepping herself; and on the other, she cannot forget the person is disabled. If she does, she may make impossible demands in performance. With the behavior strategy unresolved, the initial phase of the interaction is often quite formal with stereotypical, inhibited, overcontrolled behavior exhibited by the ablebodied participant (Kleck, Ono \& Hastorf, 1966; Kleck, 1975).

Rules of sociable interaction stipulate that both participants remain oriented toward the whole person and avoid fixing concern on any single attribute (Davis, 1961). When interacting with a disabled person, the ablebodied individual often focuses her attention on the disability, as when effortful concentration is devoted to engage or "ignore" the disability. To override this tendency, one must act as if one were dealing with the total person. The very act of disguising the focal point heightens one's awareness of the 
disability. To help ease the apparent strain, the disabled communicator feels she must also disguise her own awareness of the other's unease, thereby increasing the stress.

There is also the possibility of an incongruency of emotions evoked in the ablebodied person as she interacts with a disabled person (i.e., fear, pity, repugnance, avoidance) with the socially acceptable facial display (Davis, 1961; Goffman, 1963; Kleck, 1975). This incongruency of emotions felt and of facial display may create a heightened awareness of one's own behavior and generate an increase of unease and stress. This could account, in part, for some controlled and inhibited interaction. Also, partly controlled facial displays and termed blends may result and be confusing to the disabled partner (Ekman \& Friesen, 1979).

Davis (1961) found there is frequently an incongruency in the perceived attributes of the disabled individual by the ablebodied participant. This could create a contradiction in the way the disabled person is thought to be and the way she is seen. Frequently heard statements such as, "You are very pretty for someone crippled" supports this thesis. These contradictions are often resolved by assimilating or substituting other traits thought to be in balance with the perception of a disabled person (Heider, 1958; Davis, 1961). For example, it is often believed someone who is disabled has gained a sixth sense or has more understanding or tolerance of others. 
Besides avoiding interactions with disabled persons, ablebodied individuals often behave in other inappropriate or extreme manners (Davis, 1961; Goffman, 1963; Kleck, 1966). On occasion, over staring-behaviors toward the disabled person are observed. Frequently the ablebodied individuals feel free to interact unsolicitiously with persons who are disabled. "The implication is that the stigmatized individual is a person who can be approached by strangers at will, providing only that they are sympathetic to the plight of person of his kind." (Goffman, 1963, p. 16)

Implications of Previous Research: A Heuristic Model

With the increased anxiety felt by ablebodied persons during TADs, it could be predicted that ablebodied participants tend to self-monitor their behavior more than in TABS. Extreme self-monitoring would also account for the more formal, inhibited interactions exhibited by ablebodied communicators during TADs. One might construe this as overregulation, which is a frequent response to increased anxiety.

As the interaction moves beyond the beginning phase, it has been found that the ablebodied person tends to remain uncomfortable (Kleck, 1969; Thompson, 1982). It is different in TABS (Berger, 1975). As uncertainty is reduced, the probability that the relationship will continue 
increases. Goffman (1963) believes the maintenance, or acceleration, or discomfort in TADs is due to the fear that observers will transfer the stigma of the disability onto the other. The other will then be somehow associated, to some degree, with the disability, being labeled with the discrediting attributes of the stigmatized individual.

This maintenance, or acceleration, of discomfort could also be seen as the result of hyper-self-monitoring activity by the ablebodied communicator. Heightened awareness of the stress and of one's own behavior during the interaction could result in a loss of information that would normally be used to reduce uncertainty in TABs. A recursive loop could be created.

For example, an ablebodied woman is uncomfortable in an encounter with a disabled stranger. She is uncertain as to what are appropriate behaviors and expectations in the interaction so, she monitors her own behavior more extensively. For example, she pre-plans and analyzes her behavior, such as avoiding the use of the word "walk." She constrains her remarks to topics she perceives as safe, such as the "weather" or "how the wheelchair works." In this preoccupation with her own behavior, she fails to obtain the very information about the other that normally attends interaction. She fails to notice other specific verbal and nonverbal information. 
Essentially, her attention is focused on the disability and on her own behavior; she finds herself remaining uncomfortable as the interaction proceeds. This increases her anxiety. She wants to behave correctly for herself and the disabled person who is "less fortunate" than herself. Therefore, her self-monitoring activity accelerates. She must change the script previously tried in hopes of reducing her stress. This process creates a still greater amount of anxiety that is continued throughout this interaction and subsequent encounters.

All of the extreme behaviors and perceptual differences found in previous research comparing TADs to TABs could be a result of uncertainty as to what are appropriate behaviors where no standardized script is available. This uncertainty would increase one's self-monitoring activity (Berger \& Douglas, 1982). The implication of the studies comparing TADs to TABS is that uncertainty is greater during the former (Dahnke, 1983). The research on the processes of uncertainty reduction and attribution reports that certain information is obtained during or before (if possible) the interaction to be utilized by each person for the reduction of uncertainty. For example, one person might use the other's accent or intonation to draw inferences about the other.

Based on the above research of previous work on uncertainty reduction, attribution theory, self-monitoring 
activity, and on communication with disabled persons, the following model is proposed: (see Figure I).

Figure 1 is a heuristic model in which capital letters A through E identify processes representing the primary theoretical variables in the model. Since each process so identified subsumes a complex of interacting cognitive operations functioning through time (though rapidly), it is more realistic to construe these variables as processes corresponding to the global definitions as follows:

A. Perception of Disability/Dissimilarity. Those cognitive operations that register associations of physical difference, in this case, presence of a physical disability. Others could include race, body size, and sex, although the previous research and structural elements undergrounds this particular model are focused on physical disability.

B. Level of Self-Monitoring Activity. Amount of cognitive activity devoted to self reference, self-consciousness, and monitoring of self behavior during interaction. Cognitive involvement resulting from experiencing the disability as something to be "dealt with," as in controlled avoidance in gaze and language, active engagement in gaze and language, or other behavioral objects of self-control. 


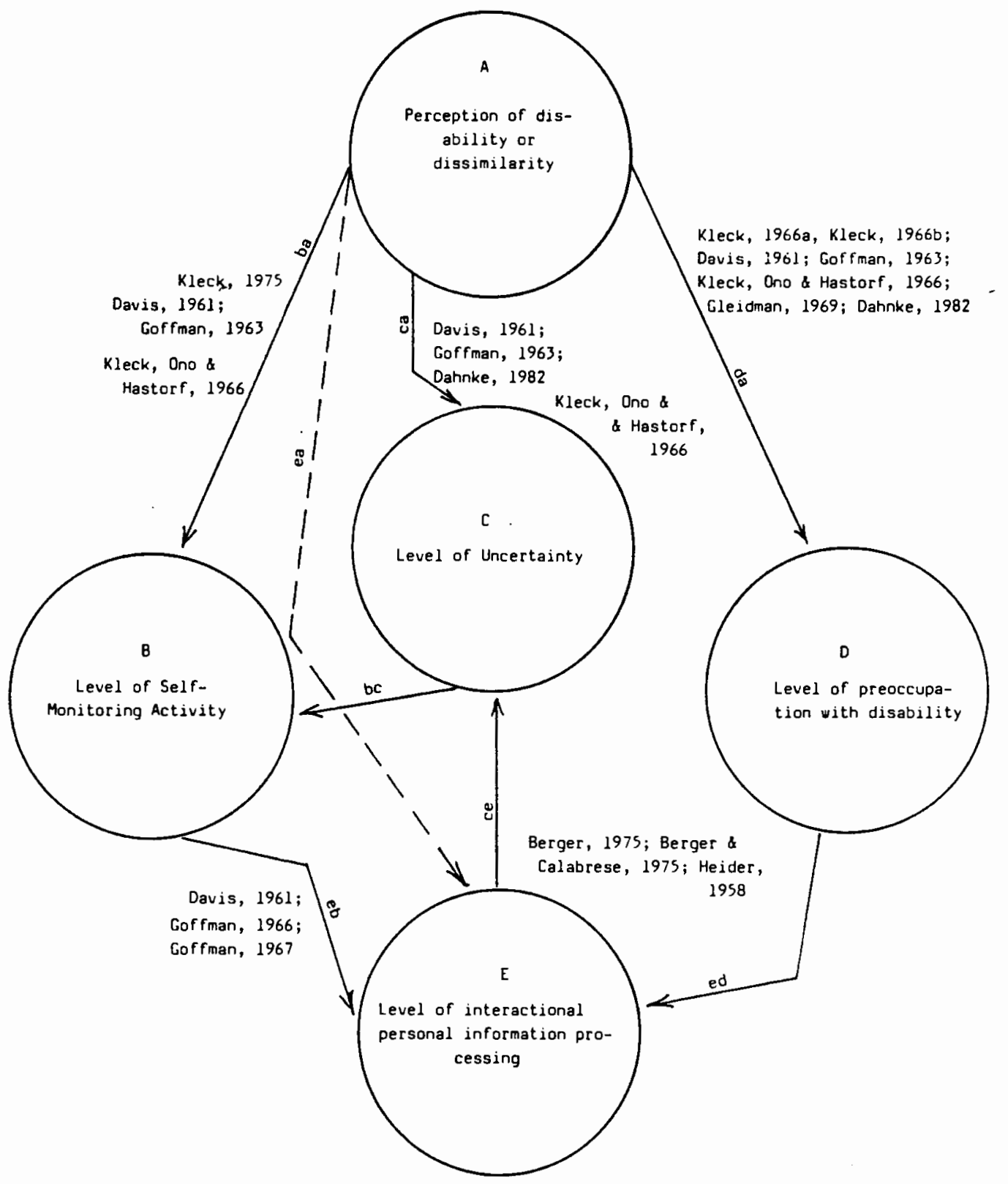

Figure 1. Heuristic model of uncertainty reduction process of ablebodied person during interaction with a disabled person. 
C. Level of Uncertainty. Amount of felt interaction strain, performance anxiety, indecision, and the need for care and caution.

D. Level of Preoccupation with Disability. Cognitive involvement with the disability part of the other's persona relating to the generally experienced distractive effects of the disability as a difference.

E. Level of Personal and Interactional Information Processing. Processing the normal interactional and other/personal content of conventional conversation, including data about the interaction as it unfolds and data about the other normally available through selfdisclosure and nonverbal interpretation. Corresponding to the language of casual modeling, the relationships among the variables in the model consist of recursive linkages denoted by 1 ines with directional arrows indicating the direction of effects and represented by a pair of lower case letters corresponding to the pair of variables so connected. Variables are connected to one another through their simple and compound paths. For example, the effect of $A$ on $B$ is a sum of the direct effect through the simple path ba and the indirect effects through the two compound paths, $c a x$ bc and da $x$ ed $x$ ce $x$ bc. One other feature of the model meriting attention is the recursive loop (BEC) comprised by the closed system of paths linking variables $B, E$, and $C$. 
An extended example is necessary to elaborate the relationship in the model. Scarlet, an ablebodied woman, perceives the disability (A) of the person with whom she is interacting. The immediate effect is an increase in her level of self-monitoring activity (B), her uncertainty level (C), and her preoccupation with the disability (D), itself. That is, heightened stress and the need for caution in dealing with the disabled individual generate higher levels of self-regulation -- in this case, increases in self-monitoring activity (B). Dealing with difference (the disabled person) directly increases scarlet's uncertainty (C) as compared to dealing with familiar entities (able-bodied persons). Preoccupation with the disability (D) as a prominent feature of the other's persona, may occupy scarlet's thoughts -- for example, to engage the disability or "ignore" it.

Subsequently, several other processes are triggered. First, with increases in self-monitoring (B), less attention is devoted to processing important personal and interactional information (E), which in turn results in less overall information and further increase in uncertainty (C). With the increase in uncertainty, scarlet monitors self activity (B) even more, which further reduces concentration on the interactional data (E) of the conversation. This proposed recursive loop (BEC) in the model could explain a number of research findings discussed heretofore, 
including why early termination of TADs occurs. Uncertainty and stress could reach unbearable levels in a very few moments of interaction through such a process. Second, with the direct increase of uncertainty (C) through the direct path ca, scarlet increases her level of self-monitoring activity (B) to still higher levels, entering fully into the BEC loop. Third, similar to increased self-monitoring activity (B), increased attention to the disability (D) also detracts from scarlet's ability to focus on and process important information (E) in the conversation, which further increases uncertainty (C), thereby adding a third engine for driving the BEC loop.

This model was developed to provide a framework for assimilating previous research on communication with disabled people, on related theoretical portions of work on uncertainty reduction and attribution, and to clarify the intent of the present study. While assessment of the total model is well beyond the scope of the present investigation, selected variables and relationships in the model will be probed to obtain empirical evidence bearing on narrowly circumscribed segments of the model. Hypotheses corresponding to three such probes will now be presented:

Hl: In interactions with a disabled person, there will be a negative correlation between the amount of self-monitoring activity by the ablebodied 
person and her level of interactional and personal information processing.

With respect to the model, this hypothesis tests the simple path eb.

H2: Able-bodied persons will engage in more self-monitoring activity during an interaction with a disabled person than during an interaction with an ablebodied person.

With respect to the model, this hypothesis assumes the C process. It tests for the direct effect of the simple path ba and the indirect effects of compound paths ca $x$ bc and da $x$ ce $x$ bc.

H3: Able-bodied persons will attend to informational sources of uncertainty reduction less during interactions with a disabled person that during an interaction with an ablebodied person.

Using the model, this hypothesis assumes $A, C$, and D processes. It tests the non-linkage ea for indirect evidence of the compound paths ba $x$ eb, ca $x$ bc $x$ ed, and da $x$ ed. 


\section{CHAPTER IV}

\section{METHODS}

The goals of this research are 1) to propose an explanatory model based on accumulating evidence that ablebodied communicators are less successful in reducing uncertainty in an interaction with a disabled person than with an ablebodied partner and 2) to conduct three exploratory probes of that model. Data on interactive behaviors with both an ablebodied and a disabled partner were obtained from subjects and coders in order to ascertain what differences, if any, were apparent in the effectiveness of uncertainty reduction in those types of conversations. The methods and procedures utilized in this study will be elaborated in this chapter. Overall design of the investigation included the following six aspects. Model development and hypotheses were discussed in previous chapters. Items two through five (below) will be discussed after a brief treatment of variable definitions and a procedural overview.

1. Development of the model and hypotheses.

2. Recruitment and videotaping of subjects.

3. Training of confederates.

4. Instrumentation.

5. Interrater reliability.

6. Data analysis and discussion. 
The problem under investigation in this study was whether or not there is a difference in acquisition of personal and interactional information in interactions with disabled strangers versus ablebodied strangers.

Definitions of Theoretical Variables

The following definitions will clarify primary variables in the hypotheses central to the experimental design and methodology of this study.

Ablebodied individual. A person who has no physical limitation that would affect mobility or dexterity. Visible, physical disability. A person who uses a wheelchair for mobility.

Self-monitoring activity. A preoccupation with one's own behavior during an encounter.

Personal and interactional information. Verbal and non-verbal information which is used to draw inferences about the partner and the interaction.

Perception of differences/disability. The belief that the other is physically disabled.

Procedural overview

Subjects of this study were involved in separate five minute conversations with two confederates, serially. Interactions per subject were conducted back-to-back, one involving an encounter with an ablebodied confederate (TAB conversations), and one with a confederate role-playing a 
disabled person in a wheelchair (TAD conversations). Seven (7) trained confederates were assigned as conversational partners to pairs of naive ablebodied subjects. Each confederate conversed from a wheelchair with one member of each pair assigned at random to the "disabled" interaction. All subjects and confederates were female. Each interaction was recorded by a video camera on the other side of a one-way mirror. Immediately following each conversation session, subjects were taken to a separate room to complete three questionnaires. All questionnaires were coded for subject, repeated measure treatment (TAB, TAD), and order of occurrence (first/second).

Subsequently, trained coders viewed the videotaped data and marked on a coding sheet each time a behavior occurred that corresponded to items on three questionnaires. These data were used to score subject's questionnaire responses as "correct" or "incorrect" recall. These recall data were entered into a data-file, and all data analyses were assisted by subprograms from Nie and others' Statistical Package for the Social Sciences (1975) on a Honeywell 6640 computing system. Based on the review of literature on uncertainty reduction, attribution theory, self-monitoring activity, and interactions with disabled persons, the model was developed and model-probing hypotheses were established. 
Recruitment and Videotaping of subjects

Twenty-four (24) naive female subjects were solicited from undergraduate courses offered by the Department of Speech Communication of Portland state University. Of the twenty-four subjects, two were eventually disqualified due to technical difficulties with the video camera which rendered the taped segment insufficient for coding purposes, three subjects were used in the pilot study, and data on nineteen additional subjects were collected for the final study. All subjects were volunteers and twenty percent (208) of the subjects were offered one credit for participation in this research. The selection of subjects represented a wide cross-section of university majors.

Each subject was told she would be assigned to two conversations in a study designed to better understand how strangers interact in initial encounters, and that she would be paired at random with two other subjects also drawn from different sections of the same course. They were informed the conversations would be videotaped and that there were three questionnaires to complete for each conversation. Before each subject was allowed to participate in the study, she was assured of anonymity and asked to read and sign a release form (see Appendix A).

The subject was taken into a small room which had been set up for videotape monitoring. The room had a one-way 
mirror on the wall opposite the only door into the room and a video camera was aimed through this mirror into the room. A microphone was installed in the room and placed on a chair in order to minimize noise other than that of the conversations. Two chairs were placed in the room, with taped markings on the floor to indicate where the back legs of the chairs were to be placed in order to remain inside the camera range. The subject was seated in the chair located in the corner of the room and was told to wait for her first partner.

While the subject was being seated in the room, the confederate was looking through the one-way mirror to determine if the subject was known to her. If not, the confederate was taken into the monitoring room, introduced to the subject, and treated as another naive subject. If the subject was known to her, another confederate was asked to replace her in that particular session. Such shuffling of confederate assignments was necessary for only one pair of forty-four (44) conversational episodes. The confederate was told to place her chair on the taped markings located in front of the entrance door after the door was closed. Both participants were reminded that their conversations would last five minutes and that both could be videotaped. Each was told to have any type of conversation that was wanted and that the study was examining the beginning phase of conversations between strangers. After 
shutting the door, the video camera in the adjacent room was started and a stop watch was used to time the conversations.

At the end of five minutes, the camera was shut off and the participants were informed the conversation was completed. The subject was asked to remain in her chair until her second partner was brought to the room and, in the presence of the subject, the confederate was asked to wait in another room for her "second" interaction. The second conversation followed the same procedure as the first for each subject.

After completing the second conversation, the subject was asked to move to another room to complete the questionnaires. She was then given two sets of questionnaires, one for the first conversation and one for the second conversation. Subjects were assured of privacy and anonymity of their questionnaire responses. Each was told to answer items without a great deal of strain, but to respond with her overall impression of the conversations. When the questionnaires were completed, subjects brought them to the video camera room. The questionnaires were then marked as described in "Procedural overview" in order to identify treatment condition, order of occurrence, and the corresponding taped segment. 
Training of Confederates

Seven (7) graduate students in the Department of Speech Communication at Portland state were selected from a pool of volunteers to act as confederates for this study. only female confederates were used in order to eliminate the gender variable. All confederates were ablebodied individuals who were trained to role-play a disabled person in a wheelchair, thereby enabling them to act as their own control in a repeated measures design.

Each confederate attended a training session in order to learn how to use a wheelchair and of possible postural attitudes of disabled persons. For example, each confederate was told to be aware of foot and leg movements while role-playing in a disabled mode. They were trained to enter a doorway into a room while seated in a wheelchair. Each confederate practiced movement of the wheelchair until she was confident in turning corners, entering rooms, and stabilizing the wheelchair. Confederates were told to enter each conversation as if it were their first interaction, and were cautioned to behave in all other details as they did in their TAB experimental sessions.

\section{Instrumentation}

Development of questionnaires. Three questionnaires were developed for subjects to complete immediately following each set of interactions. The first questionnaire, 
Partner's Appearance and Non-verbal Behavior (PANB), consisted of eleven (11) questions regarding the other's appearance and non-verbal behavior (see Appendix B). Subjects (S's) were asked to respond to items such as the length of the other's hair, the type of shoes worn by the other, and the amount of gestural activity. The purpose of this questionnaire was to test S's use and recall of available visual information from the partner. These data comprised one part of the operational definition of "level of personal and interactional information," represented by Variable $\mathrm{E}$ in the model (p. 26).

The second questionnaire, Partner's Vocal and Verbal Behavior (PVVB), consisted of ten (10) questions regarding the other's vocal and verbal behavior (see Appendix $C$ ). For example, the subject was asked to recall how often the other changed topics, how many verbal agreements the other exhibited, and to recall the rate and loudness of her partner's vocalizations. This questionnaire was developed to measure S's use and recall of available auditory information from the partner. These data comprised the second part of the operational definition of "level of personal and interactional information," represented by variable E in the model.

The third questionnaire, self Behavior (SB), consisted of twelve (12) questions pertaining to the subject's own verbal and motor behaviors (see Appendix D). For example, 
the subject was asked to recall how often she registered agreement during the conversation. The purpose of this questionnaire was to index S's level of self-monitoring activity, and comprised the operational definition of "selfmonitoring activity," represented by variable $B$ in the model.

No SB items were unique. Four (4) duplicated PANB items and seven (7) items duplicated PVVB items. Therefore, the three questionnaires were comprised of twenty-two (22) unique items in all. Coding trials, discussed in a subsequent section, reduced this total to nineteen (19) total scorable items. In addition, an item not scorable in the usual manner was included on the SB and PANB. This guestion asked S's to recall the non-verbal behavior employed by partner and self, respectively, during the conversation and was merely totaled for each conversation. Training of Coders and Content Analysis of Videotapes. Three (3) students from Portland state University were trained to code aspects of videotaped conversations. They were told there would be twenty-two (22) taped pairs of conversations totaling forty-four (44) conversations, each involving one subject and one confederate interacting together. Coders were to watch each conversation twice, once to score the subject in the dyad, and once to score the confederate. They were to record each time a behavior 
corresponding to questionnaire items was observed (see Appendix E). For example, each gesture that S's made was to be recorded, each verbal agreement heard was to be recorded, and each subject-initiated topic change was to be marked. The coders then reviewed this taped segment and recorded C's behavior in the same manner. The coding forms were then marked to identify treatment condition, order of occurrence, and the corresponding taped segment.

Coders watched a practice conversation to gain skill in noticing behaviors which corresponded with the questionnaire items. After several attempts, the coders felt confident to begin testing for interrater reliability.

To test for interrater reliability, the three coders watched the first three pairs of conversations and coded the taped data. They were then asked to view the remaining tapes assigned as follows. Coder 1 scored all twenty-two (22) pairs of conversations. Coder 2 scored numbers four (4) through thirteen (13). Coder 3 scored numbers fourteen (14) through twenty-two (22). This procedure produced criterion data which, after transferring to data forms, were used to score questionnaire responses.

Transferred Coder Data to Data Forms. With the coded records of subject and confederate behaviors in hand, it was then necessary for the investigator to convert coder's scores from coding forms into the response categories on various questionnaire items. Data forms following the 
questionnaire and coding sheet format were used for this purpose (see Appendix F). For example, questionnaires contained items having response categories with either ratio level (frequency) or ordinal (rank order) ingredients. Such questions as "How often did your partner interrupt you?" had frequency categories of "0-2 times," "3-5 times," and "over 5 times." The ordinal response items employed category descriptions of "very little," "a moderate amount," and "a great deal."

Frequency response items asking for a specific number were easily transformed by noting the exact frequency of occurrence of each behavior observed by coders from the videotaped data. If a behavior was noted twice, it was coded as "0-2 times;" if a behavior was noted four (4) times, it was coded as "3-5 times." Any behavior noted over five (5) times was coded in the "over five times" category.

The ordinal response items were scored in the following way. Behaviors which were noted five times or less were classified as "very little." Behaviors observed by the coders in amounts between six (6) and fifteen (15) were classified as "a moderate amount." Any behaviors which were observed by coders over fifteen (15) times were classified as "a great deal." These answers were then marked on data forms. As with the original questionnaires and the coding forms, the data forms were marked to identify 
treatment conditions, order of occurrence, and the corresponding taped segments.

Scoring Questionnaire Data. After the data forms were completed by the investigator for each conversation, these data were compared to S's responses on the corresponding questionnaires permitting each S's questionnaire response to be scored "correct" or "incorrect" recall for each item. Each correct answer received a "1." Each incorrect recall was coded with a "0." Correct recall responses were totaled for each questionnaire per subject.

It was immediately apparent there were three questions which needed to be eliminated from the study. Question 1 on PANB, which asked "What was the color of your partner's hair?" was eliminated due to technical difficulties of color contrast on the taped segments. Filming through the one-way mirror made subtle hair color distinctions too difficult for coders to assess.

The second and third questions to be eliminated were question 2 on PVVB and question 6 on SB which asked S's to identify topics initiated by their partners and themselves. It was found to be too difficult for coders to identify the specific topics. All other items were found to be amenable to straight-forward interpretation and, as reported below, highly reliable. 
Interrater Reliability

A pilot study was conducted to test the workability of the experimental design and to examine the interrater reliability between coders and content analysis of taped conversations. The reliability study entailed three coders independently performing content analysis on six (6) conversations with respect to all content defined by questionnaire items. A pair of five (5) minute taped conversations from each of three (3) subjects were used for analysis in this pilot study. For three of these conversations, coders scored Ss; in the second three, they scored Cs. These conversations were chosen from the first few videotaped sessions. Thus, the pilot study data involved three (3) coders and consisted of eighteen (18) content analyses of six (6) conversations.

Coder Agreement on Raw Data. As described in "Training coders and content analyses of videotapes," coders performed content analyses of videotaped conversations by recording frequency of occurrence for behaviors corresponding to all questionnaire items. Thus, frequency data were obtained for all behavior corresponding to every questionnaire item, regardless of the response category format (numerical or adjective), of a particular questionnaire item. There were ten (10) items with numerical response categories. These included items on gesture, facial 
expression, topic change, vocal pauses, personal and impersonal self-disclosure, interruptions, vocal agreement, questions, and vocal variety. Of these ten (10) items, vocal variety and interruptions generated 100 percent agreement among all three coders. With the exception of one item, the largest raw numerical discrepancy between any of the three coders for any given item was "2."

The adjective response category items included eye contact, posture, vocal volume, rate of speaking, type of shoes, type of clothing, length of hair, body type, and whether or not the confederate wore glasses. All three coders agreed with absolute numerical precision in fiftyeight (58) percent of their judgments. With the exception of a single judgment trial on one item in one conversation, the remaining forty-two (42) percent of coders' judgment resulted in a discrepancy of two-or-less in these frequency of occurrence data.

Coder Agreement on Category Data. The above results achieved precision well beyond what was required here. Coders' raw frequency data were subsequently collapsed into three categories per item to correspond to judgments which S's could reasonably be expected to make; viz. the threefold numerical and adjective response categories of all questionnaire items.

After all coding forms were completed with the coders' frequency tabulations, data were transposed to numerical or 
adjective questionnaire categories as described in a previous section. For the former items, coders' raw data were classified on the basis of the precise numerical response limits of the questionnaire response categories. For the latter, coders' frequency scores were classified using category limits described in the section on "Transferring coder scores to data forms."

After placing all coder responses into these categories, 100 percent agreement among all three coders was obtained for the pilot study. For the remaining nineteen (19) conversation sets in this study, coders failed to agree within the interval level and ordinal categories less than nine (9) percent of the time. In cases of disagreement between two coders, the investigator reviewed the tape segment and made the judgment. The investigator acted as referee in 119 individual items out of 1320 total coded responses. The remaining 91.15 percent of coder judgments represented complete agreement and did not require arbitration.

Coding forms were completed by two coders for each conversation as described in section "Training of coders." Following the procedure described in "scoring Questionnaire Data," the investigator completed data forms for all conversations and S's questionnaire responses were marked as correct (1) or incorrect (0). Subsequently, these data along with individual questionnaire totals were entered into a 
data-file through the Honeywell 6640 computing system for analysis. All data analyses were performed with the assistance of the "PEARSON CORR" and "T-TEST" subprograms of Statistical Programs for the Social sciences (Nie et al, 1975). 
CHAPTER $V$

RESULTS

Two separate statistical procedures were applied to the data on a Honeywell 6640 computing system. "T-TEST: and "PEARSON CORR" subprograms of the Statistical Packages for the Social Sciences (Nie and others, 1975) were used to assess the three hypotheses of the present study. The 05 alpha level was the criterion applied to all statistical tests. All hypotheses were directional, and one-tailed tests were therefore applied to outcomes that were in the predicted direction. In some cases, results were contrary to predicted directions, and in those cases, two-tailed tests of significance were used.

\section{Hypothesis I}

Hl: In interactions with a disabled person, there will be a negative correlation between the amount of self-monitoring activity by the ablebodied person and her level of personal and interactional information processing.

At the outset, it was expected from previous work on uncertainty reduction, attributional processes, self-monitoring activity, and transactions between ablebodied and disabled persons, that the TAD mode of interaction would 
operate to increase the level of self-monitoring activity. The increased self-monitoring activity would, in turn, reduce the level of personal and interactional information processes in TAD's, resulting in a negative correlation between self-monitoring levels (SB) and information about partner (PANB and PVVB). This, however, was not the case. Using subprogram "PEARSON CORR," a Pearson productmoment correlation coefficient was computed in order to assess the direction and strength of the relationship which might exist between self-monitoring activity and interactional information recall in all TAD conversations. Questionnaire totals. Contrary to Hypothesis I, correlations of questionnaire totals between self-monitoring activity and partner nonverbal and verbal information recall achieved positive values, +.275 and +.118 , but failed significance at $p=.108$ and $p=.30$, respectively. Matched pair analysis. As described in the "METHODS" chapter, some questionnaire items were unique to the PANB and PVVB questionnaires. The remaining items on those two questionnaires had identical "companion" items on the SB questionnaire. Since the various items tapped recall of qualitatively different behaviors, it was felt an examination of the correlation of those matched pairs would provide content specific tests of the first hypothesis. A correlational analysis of the ten matched items was performed, and the results are listed in Table I. 
TABLE I

PEARSON CORRELATION ON TEN MATCHED PAIR ITEMS ASSOCIATED WITH SELF-MONITORING AND INFORMATION RECALL IN INTERACTIONS WITH DISABLED PEOPLE

\begin{tabular}{lcc} 
VARIABLE PAIR & $\mathrm{r}$ & $\mathrm{p}$ \\
\hline SB1-PANB6 & $.4166 \mathrm{a}$ & .054 \\
SB2-PANB7 & $.4368 \mathrm{a}$ & $.042 *$ \\
SB3-PANB8 & -.0925 & .341 \\
SB4-PANB9 & $.0636 \mathrm{a}$ & .750 \\
SB5-PVVB1 & $.6236 \mathrm{a}$ & $.003 * \star$ \\
SB6-PVVB9 & $.1845 \mathrm{a}$ & .412 \\
SB7-PVVB2 & -.0430 & .425 \\
SB8-PVVB4 & $.0953 \mathrm{a}$ & .674 \\
SB9-PVVB3 & $.0129 \mathrm{a}$ & .954 \\
SB10-PVVB5 & -.0169 & .470 \\
\hline
\end{tabular}

$* \mathrm{p}<.05$

$* * \mathrm{p}<.01$

a These coefficients carried signs opposite to that predicted of the hypothesis and therefore, were assessed by two-tailed tests.

As predicted in the first hypothesis, Table I shows the negative correlations between self-monitoring activity and interactional information in three individual items, which include gestural activity (SB3), self-disclosure (SB7), and agreement (SB10), respectively. Although these items obtained a negative correlation, they failed to 
achieve significance. Contrary to this hypothesis the remaining seven items registered a positive correlation between self-monitoring activity and information recall, two of which were at statistically significant levels. These items include posture (SB2), and topic change (SB5).

Nonverbal behavior recal1. As described in the "Methods" chapter, items SBII and PANBI0 were coded differently from other items. These two questionnaire items asked S's to 1 ist recall of specific nonverbal behaviors of self and other. Each item was then scored and coded according to the total amount of correct recall. It was expected that recall of nonverbal behavior of self would register higher levels during the TAD treatment condition while recall of partner would decrease, resulting in a negative correlation between self-monitoring activity (SB) and information processes (PANB). A correlational analysis on the two items was performed and, contrary to the first hypothesis, a positive correlation of +.6435 was obtained, significant at $p=$ .033 .

Follow-up data analysis on TABs. Beyond the scope of Hypothesis I, data in hand from TABs provided an opportunity to replicate results from previous studies on the association between self-monitoring activity and communication competence. That is, during normal (e.g., ablebodied) conversations, previous work shows higher levels of selfmonitoring activity was associated with proficiency in 
awareness of information and adjustment to the partner (snyder, 1974). Since such awareness is indexed by PANB and PVVB data, one would expect a positive correlation between those measures of self-monitoring activity and informational processes in the data associated with the ablebodied mode of interactions. Therefore, a correlational analysis was performed on questionnaire totals (SB/PANB and SB/PVVB) in the TAB treatment condition. These produced positive correlation between self-monitoring and personal and interactional information processes in all but two items, and the results are listed in Table II.

Previous work on the association of self-monitoring activity and communication competence predicted that there would be a positive correlation between SB and PANB/PVVB items. This was the case in eight out of ten items. Statistical significance was achieved in one item, vocal pauses (SB6). The remaining seven items which obtained positive correlations were not statistically significant. Contrary to previous work on self-monitoring activity and communication competence, two of the items there were found to have a negative correlation; however, both were of negligible magnitude. These items are facial expression (SB4) and self-disclosure (SB7). 


\section{TABLE II}

FOLLOW-UP CORRELATION ON TEN MATCHED PAIR ITEMS

ASSOCIATED WITH TABS IN INTERACTIONS

WITH ABLEBODIED PARTNERS

\begin{tabular}{lcc} 
VARIABLE PAIR & $\mathrm{r}$ & $\mathrm{P}$ \\
\hline SB1-PANB6 & .2797 & .104 \\
SB2-PANB7 & .0925 & .341 \\
SB3-PANB8 & .2774 & .106 \\
SB4-PANB9 & $-.1604 \mathrm{a}$ & .238 \\
SB5-PVVB1 & .2951 & .091 \\
SB6-PVVB9 & .4667 & $.014 *$ \\
SB7-PVVB2 & $-.1207 a$ & .296 \\
SB8-PVVB4 & .3269 & .069 \\
SB9-PVVB3 & .1604 & .238 \\
SB10-PVVB5 & .1658 & .230 \\
\hline
\end{tabular}

$* \mathrm{p}<.05$

a These coefficients carried signs opposite to the prediction of the hypothesis and therefore, were assessed by two-tailed tests.

Hypothesis II

H2: Ablebodied persons will engage in more self-monitoring activity during an interaction with a disabled person than during an interaction with an ablebodied peer.

From previous work on self-monitoring activity and interactions between ablebodied and disabled persons, it was expected that self-monitoring activity would register 
higher levels during TADs as compared to TABs, resulting in a significantly larger mean for TAD data. This was not the case. For this hypothesis, "t" tests were used to assess the difference between means of the TAB and TAD conversational treatment condition for self-monitoring activity.

Questionnaire totals. The second hypothesis predicted that self-monitoring activity (SB totals) for the TAD treatment condition would register greater means than in the TAB treatment condition. Contrary to this hypothesis, the outcome showed a greater mean in the TAB treatment condition with a $t$ value of 1.11 , but failing significance at $\mathrm{p}=.274$ as shown in Table III.

TABLE III

T-TEST ON SB TOTALS ASSOCIATED WITH TAB

AND TAD TREATMENT CONDITION

\begin{tabular}{lcccc} 
VARIABLE & TABX/TADX & S.E. & $t$ & P \\
\hline \multirow{2}{*}{ SBTOTS } & $6.0000 / 54091$ & .526 & 1.11 & .274 \\
\hline
\end{tabular}

Matched pair analysis. For reasons previously discussed under Hypothesis $I$, an analysis of ten matched pairs of $\mathrm{SB}$ versus PANB/PVVB items was conducted. Several " $t$ " tests were performed on the ten matched items, and the results are listed in Table IV. 
T-TEST ON TEN SB ITEMS ASSOCIATED WITH

TAB AND TAD TREATMENT CONDITIONS

\begin{tabular}{ccccc} 
ITEM & TABX/TADX & S.E. & $t$ & $P$ \\
\hline 1 & $.5455 / .7727$ & .130 & 1.74 & $.048 *$ \\
2 & $.5909 / .3636$ & .091 & $2.49 \mathrm{a}$ & $.030 *$ \\
3 & $.5909 / .4091$ & .125 & $1.45 \mathrm{a}$ & .162 \\
4 & $.4545 / .3636$ & .146 & $0.62 \mathrm{a}$ & .540 \\
5 & $.6364 / .5455$ & .130 & $0.70 \mathrm{a}$ & .492 \\
6 & $.3182 / .3636$ & .080 & 0.57 & .288 \\
7 & $.3636 / .1818$ & .125 & $1.45 \mathrm{a}$ & .162 \\
8 & $.5909 / .6818$ & .130 & 0.70 & .246 \\
9 & $.4545 / .3182$ & .119 & $1.14 \mathrm{a}$ & .266 \\
10 & $.3636 / .5455$ & .142 & 1.28 & .106 \\
\hline
\end{tabular}

${ }^{*} \mathrm{p}<.05$

a These mean differences were in the opposite direction to that predicted by the hypothesis and therefore, were assessed by twotailed tests.

As predicted in Hypothesis II, mean differences in self-monitoring activity were larger in the TAD conversational condition than in TABs for four questionnaire items. These items include eye contact (SBI), vocal pauses (SB6), self-disclosure (SB8), and vocalized questions (SBI0). SBI was statistically significant at $p=.048$. Contrary to the second hypothesis, mean differences for self-monitoring 
activity were larger during the TAB treatment condition than during TADs in the remaining six questionnaire items, with posture recall (SB2) statistically significant at $p=$ .030 .

Nonverbal behavior recall. For reasons previously discussed, item 11 from SB questionnaire was scored differently from other questionnaire items. Hypothesis II predicted that recall of one's own nonverbal behavior would be greater during the TAD treatment condition than during TABs, as indicated by a significantly higher mean for TAD data. A "t" test was computed and, as predicted by the second hypothesis, the mean was higher for the TAD data, with a $t$ value of 0.22 , but failing significance at $\mathrm{p}=.412$.

\section{Hypothesis III}

H3: Ablebodied persons will attend to informational sources of uncertainty reduction less during interactions with a disabled person than during an interaction with an ablebodied peer.

From previous work on uncertainty reduction, attributional processes, and interactions between ablebodied and disabled persons, it was expected that processing of personal and interactional information about the partner would be less during TADs as compared to TABs. As described in the "Methods" chapter, two questionnaires (PANB, PVVB) 
asked for S's informational recall of partner for both conversational conditions. Each asked recall of exclusively nonverbal (PANB) or exclusively verbal (PVVB) behavior. Each questionnaire total was assessed separately. Questionnaire totals for PANB. The third hypothesis predicted that the mean PANB score for TAB conversations would be larger than for TADs. The direction of mean difference supported this hypothesis with the TAB mean showing a larger value than for TAD mean for appearance and nonverbal recall, with a $t$ value of 0.27 , but failing significance at $\mathrm{p}=.385$ ( see Table $\mathrm{V}$ ).

Questionnaire for PVVB. As predicted in Hypothesis

III, the mean score for TABs was larger than for TADs for the PVVB totals. The mean difference for verbal information recall obtained a $t$ value of 2.04 , which was significant at $\mathrm{p}=.024$ ( see Table V).

TABLE V

T-TEST ON PANB AND PVVB TOTALS ASSOCIATED

WITH TAB AND TAD TREATMENT CONDITIONS

\begin{tabular}{llccc} 
ITEM & TABX $/$ TADX & S.E. & $t$ & $P$ \\
\hline PANB & $6.7727 / 6.6364$ & .544 & 0.27 & .385 \\
PVVB & $6.5909 / 5.5909$ & .526 & 2.04 & $.024 *$ \\
\hline${ }^{*} \mathrm{P}<.05$ & & & &
\end{tabular}


Matched pair analysis. Several " $t$ " tests were performed on individual item scores for all eighteen items of the PANB and PVVB questionnaires in order to assess mean differences between TAB and TAD conditions. The results are listed in Table VI.

As predicted by Hypothesis III, Table VI shows that mean differences favored TABs over TADs for ten of the questionnaire items. Of these ten items, PANB (facial expression) and PVVB6 (voice type) were statistically significant with a $t$ value of 1.74 at $p=.048$, and a $t$ value of 2.31 at $\mathrm{p}=.015$. Contrary to the hypothesis, a greater mean for the TAD treatment condition than for TABs was found in the eight remaining questionnaire items. None of these differences approached statistical significance.

Nonverbal behavior recall. For reasons previously addressed, item 10 from the PANB questionnaire was coded differently from the other questionnaire items. Hypothesis II predicted that recall of other nonverbal behavior would be less during the TAD treatment condition then during TABS. A "t" test was performed, and obtained a value of 1.82, statistically significant at $\mathrm{p}=.041$.

\section{Summary}

In interactions with a disabled partner, self-monitoring behavior was negatively correlated with recall of information about partner for only three of ten matched 
T-TESTS ON PANB AND PVVB ITEMS ASSOCIATED WITH TAB AND TAD CONVERSATIONAL CONDITIONS

\begin{tabular}{|c|c|c|c|c|}
\hline ITEM & TABX/TADX & S.E. & $t$ & $P$ \\
\hline PANBI & $.9091 / .8182$ & .063 & 1.45 & .081 \\
\hline PANB 2 & $.5000 / .5455$ & .154 & $0.30 a$ & .770 \\
\hline PANB 3 & $.7727 / .6364$ & .165 & 0.83 & .208 \\
\hline PANB 4 & $.6364 / .7273$ & .146 & $0.62 a$ & .540 \\
\hline PANB 5 & $.7273 / .8636$ & .119 & $1.14 \mathrm{a}$ & .266 \\
\hline PANB6 & $.8182 / .8636$ & .130 & $0.57 a$ & .576 \\
\hline PANB7 & $.5000 / .5909$ & .130 & $0.70 a$ & .492 \\
\hline PANB 8 & $.5000 / .5000$ & .174 & 0.00 & .500 \\
\hline PANB9 & $.6818 / .4545$ & .130 & 1.74 & $.048 *$ \\
\hline PVVBI & $.4545 / .3182$ & .136 & 1.00 & .164 \\
\hline PVVB2 & $.5000 / .5455$ & .139 & $0.33 a$ & .746 \\
\hline PVVB3 & $.3182 / .1364$ & .125 & 1.45 & .081 \\
\hline PVVB 4 & $.8182 / .7727$ & .123 & 0.37 & .357 \\
\hline PVVB5 & $.5455 / .5909$ & .154 & $0.30 a$ & .770 \\
\hline PVVB 6 & $.9091 / .5909$ & .138 & 2.31 & $.015 *$ \\
\hline PVVB7 & $.5000 / .3636$ & .119 & 1.14 & .183 \\
\hline PVVB8 & $.8636 / .7727$ & .112 & 0.81 & .213 \\
\hline PVVB9 & $.6818 / .7727$ & .112 & $0.81 a$ & .426 \\
\hline
\end{tabular}

$* p<.05$

a These mean differences were in the opposite direction to that predicted by the hypothesis and therefore, were assessed by twotailed tests. 
items; amount of gestural activity, amount of self-disclosure, and amount of agreement. None of those correlations was statistically significant and the remaining seven items, as well as the self-monitoring/partner information questionnaire totals, produced positive correlations, contrary to the prediction of the first hypothesis. A follow-up replication analysis of the relationship between self-monitoring and partner information in ablebodied interactions produced the expected positive correlations in eight of the ten matched items. Most correlation coefficients were negligible or weak, but one (vocal pauses) was statistically significant.

Comparisons of self-monitoring behavior in interactions with an ablebodied versus disabled partner produced mean differences in the predicted direction for four out of ten matched items. Subjects engaged in more self-monitoring in disabled interactions with respect to eye contact, vocal pauses, self-disclosure and vocalized questions; however, only eye contact generated a statistically significant difference. The direction of difference in self-monitoring for the remaining six items and for the self-monitoring questionnaire total favored the interaction with an ablebodied partner condition, contrary to the second hypothesis. The direction of mean difference in self-monitoring for the remaining six items, for the self-monitoring questionnaire total, and for a separately analyzed nonverbal 
behavior recall item favored the interaction with an ablebodied partner condition, contrary to the second hypothesis.

The third hypothesis predicted that recall of information about partner, both verbal/vocal information and appearance and nonverbal information, would be greater in interactions with ablebodied partners than with disabled partners. Questionnaire totals for both verbal and nonverbal recall supported the hypothesis with respect to direction of mean differences. In addition, the difference for verbal/vocal information recall was statistically significant. Mean differences for ten out of eighteen recall items were in the predicted direction. Mean differences for two of the ten, facial expression and voice type, were statistically significant. Mean differences in the remaining eight items were contrary to the predicted direction. Mean differences for the separately analyzed nonverbal recall item also supported the hypothesis and was statistically significant. 
CHAPTER VI

\section{DISCUSSION}

The experiments went extremely well in both the pilot and final studies. Once the videotaping error, which made it impossible to view and code two subjects, was corrected, all conversational sets were clearly seen by the coders. The overall investigation did not have to be altered in any way.

Credibility of Experiment Manipulation

One major concern of the author was the credibility of the confederates in two areas. The first concern was that repeated interactions of confederates would eliminate the spontaneity and freshness of the conversations. Before each interaction, all confederates were reminded to act as if this were their first conversation, and in all ways to behave as a naive subject. There was no indication from S's that confederates were not believed as such. Verbal reports from confederates reflected an anxiety with respect to behaving correctly upon entering into each conversation, which aided in their appearance as naive subjects.

The second concern regarding confederates was "were confederates credible as disabled individuals in the TAD conversational mode?" Three post-session subject reports 
eased this concern. Immediately following an experimental session, one confederate who had been role-playing a disabled person, realized the subject was slightly known to her as a student. The investigator immediately contacted the subject and explained the entire research project. The subject stated she had not recognized the confederate during the encounter because she had been too nervous "while talking with someone who used a wheelchair." She had failed to identify her partner, she said, because, "You're not supposed to look at people who are disabled."

Two other post-session subject reports also eased the investigator's concerns regarding credibility. Both subjects met their "disabled" partner on campus after participating in this study but, before the investigator was able to fully debrief all subjects. Both subjects approached the investigator expressing feelings of having been "tricked." subjects responded positively after a full debriefing.

\section{Limitations of the study}

There are a few limitations of this study in both the methodological and theoretical aspects. The first is the problem of volunteerism of the subjects. For example, it may be that people who volunteer to participate in research in the area of speech Communication are more motivated and competent in the communication process. This may be 
especially true of subjects selected from classes in this area of study. This limitation will be elaborated within this chapter.

All subjects chosen for this project were female in order to eliminate possible gender-related communication style differences. Studies have shown that females tend to be more accurate in the interpretation and use of nonverbal activity (Burgoon \& Saine, 1978). If males had been chosen as subjects, the results of this study may have been different. For example, male communicators may be less accurate in the use of nonverbal activity, thus may have recalled less nonverbal activity used by their partners.

The awareness that all conversations were being videotaped by both subjects and confederates may have increased the overall anxiety level during the encounters. This increase of anxiety may have created a more homogeneous climate which could have reduced differences between the transactions involving ablebodied persons and those involving disabled people.

The categorizing of the adjective response items on the questionnaires may also be a limitation. Category limits were subjectively selected by the investigator after watching twenty-two taped conversations.

To assess cognitive processes such as those examined in the three hypotheses of this study by inferring from a pen and paper recall method may be problematic. For 
example, the investigator relied on specific verbal and nonverbal behaviors, such as gestural activity or facial gaze, to tap individual responses. The questionnaire items may not have reflected the relevant specific behaviors associated with the constructs for some individuals.

Another limitation of this study may be a lack of theoretical work in the literature on a major variable of this study, specifically in the area of self-monitoring activity. For example, more research needs to be conducted on the use of self-monitoring activity and its effects on communication competence.

The attitude of subjects toward disabled people was unknown in this study. It was felt that testing individual attitudes, either before or after the experimental session, would contaminate the outcome.

This study attempted to connect many theories for the first time. For example, an attempt was made to connect self-monitoring activity with uncertainty reduction and attributional processes. From a review of the literature, the investigator believed that self-monitoring activity had a curvilinear relationship with the efficiency with which one processes personal and interactional information about one's partner. That is, although no data were available that directly tested or supported such a curvilinear hypothesis, it seemed plausible from what studies had been done, that exceedingly high self-monitoring would work to compete 
with attention to partner's characteristics, thereby reducing information about partner, during (therefore after) an interaction with a disabled partner.

The present study also attempted to initiate a basis for a theoretical model relating heretofore disconnected theories and bodies of work on diverse constructs: selfmonitoring, uncertainty reduction, attribution theory, and communication with disabled people. Isolated studies provided some clues to guide model development. For example, Kleck (1966) found that ablebodied individuals experienced a more inhibited, formal interaction during encounters with disabled individuals. Such behavior could be understood by positing an underlying and dysfunctional increase in selfmonitoring, even though other research with ablebodied individuals indicated moderate increases in self-monitoring enhanced communication awareness, adaptation to other, and overall performance (snyder, 1974).

As a rudimentary effort at model-building in this area, it is likely that some one or several critical variables were omitted or that some variables in the model were really multi-factored conglomerates of several discrete and self-canceling variables. In addition, in an effort to keep the "size" of the project under control, no data were collected on two of the five variables in the model from which the hypotheses were derived. The limitation had at least two consequences. First, multiple, and therefore 
potentially confounding, assumptions were necessary trappings of the hypotheses. Second, the more powerful and informative data analysis procedures of causal modeling/path analysis were not an option.

Notwithstanding the above several important limitations of the present study, the writer contends that this research produced some important findings. The data collection procedures were demanding and complex, but were conducted with clarity of purpose and precision. Due in part to that feature, coder reliability was commensurately high and trustworthy. The repeated measures design provided a strong basis for inference for that portion of the results that were positive. Finally, a first attempt has been made to grapple with the challenging process of building an integrated theory of communication with disabled people. Some of the matters discussed above, positive and negative, will be referred to in connection with specific hypotheses in the following sections of this report.

\section{Hypothesis I}

The correlation between self-monitoring and information recall failed to support the hypothesis. This could be due to many reasons. First, there has been no previous research which has attempted to link self-monitoring activity explicitly with the amount of information gathered by a communicator. 
In gathering research for this hypothesis, the author believed there to be implications that self-monitoring behavior was curvilinear, that both a very low self-monitoring individual and a hyper-self-monitoring individual would fail to be sensitive to the partner and the situation. The implication was that less information about the other would arise when one was hyper-self-monitoring due to a shift in the attention from the other. Thus, the first hypothesis focused on the predicted downturn part of the supposed curvilinear relationship specific to interactions with disabled people only (see Figure 2).

Not all researchers agree that an increase in selfmonitoring activity (e.g., a "high" self-monitor) will increase a communicator's competence through that communicator's becoming more sensitive to the other and the situation. For example, in the follow-up analysis on the ablebodied partner treatment in the present study, only one out of ten recalled behaviors showed statistically significant positive correlations. In this light, it could be that the theory of self-monitoring itself may need to be explored before future connections and implications can be stated. Secondly, the selected method of the study to assess the cognitive processes of self-monitoring may have hindered the predicted response in this hypothesis. Questionnaire items may not have reflected relevant information specific to self-monitoring activity. Since each person 


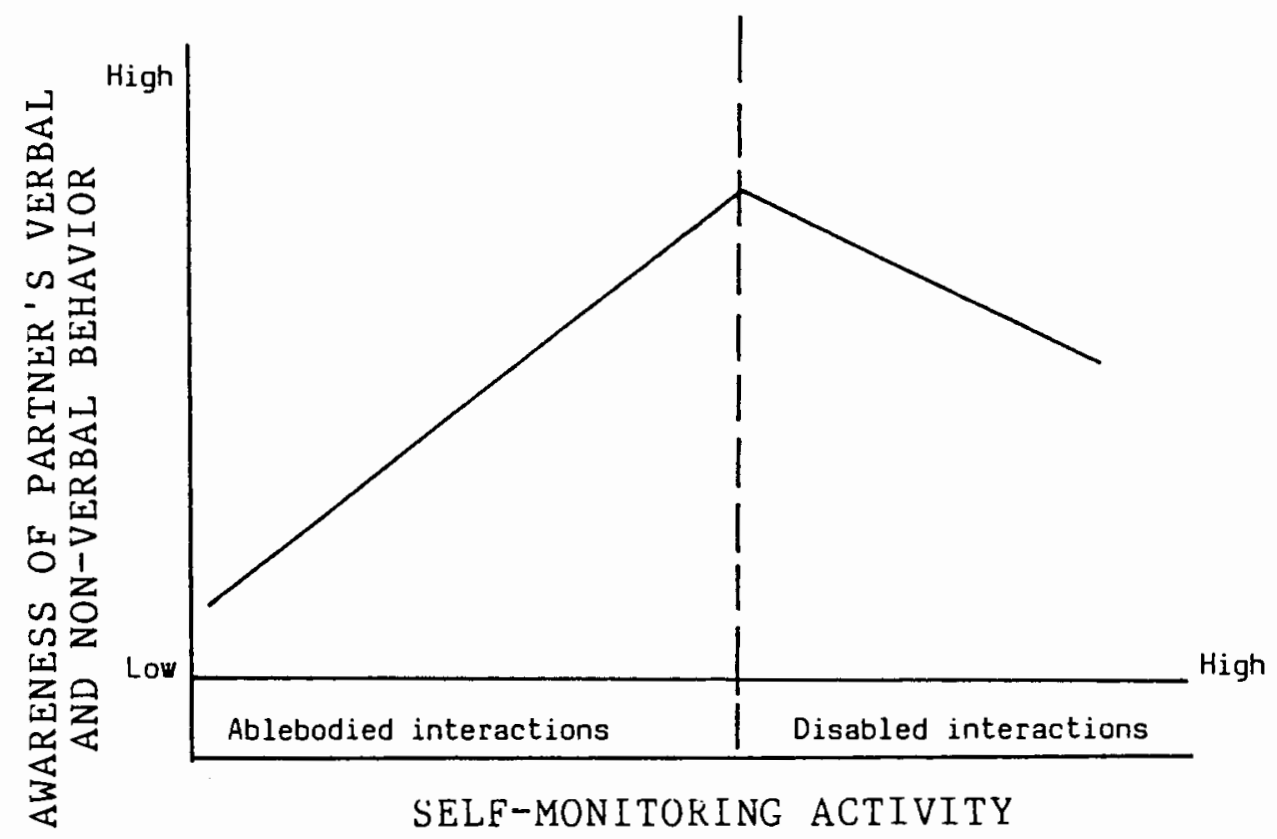

Figure 2. Relationship of self-monitoring and attention to partner behavior. 
has individualized scripts that are followed in encounters, questionnaire items may not have tapped personal specific content, germane to each subject.

In the same vein, there may have been an error in the selection of which self-recall behaviors were relevant to loss of information about the partner. An analysis of matched-pair items which tapped specific behaviors was conducted. It may be that the increased awareness of one aspect of one's own behavior, such as eye contact, resulted in the loss of information in a different aspect of partner's behavior, e.g., facial expression instead of eye contact.

By subjectively selecting category limits of less than five, between five and fifteen, and more than fifteen for adjective questionnaire items of "very little," "a moderate amount," and "a great deal," respectively, the coding protocol may have changed the outcome of the hypothesis. After viewing twenty-two taped conversations, the investigator noticed a marked difference in verbal and nonverbal behaviors at these limits within each five minute conversation. If the limits had been selected differently, the outcome of this hypothesis may have been changed. For example, if the category of "a moderate amount" had been selected as five to ten instead of five to fifteen responses, some subjects who had chosen that category would have been scored as incorrect. 
The last possible reason for the outcome to reflect a positive correlation between self-monitoring and information recall may be the subjects themselves. As addressed in "Limitations of the study," volunteers may be more motivated than those who do not volunteer to participate in research. This motivation could heighten the subject's sensitivity to the other and the situation, lending to a homogeneously high communication competence. It may be also that volunteers are often those who are already more competent and confident in their ability to communicate. In addition, subjects for this study were selected from courses in the field of speech Communication, while all confederates were speech Communication majors. The possible similarity of interests may have created greater homophily among the subjects and confederates, the totality being a heightened awareness of the self and the other (Novak, 1968).

Hypothesis II

Subjects recalled self-behavior more during the conversation with a disabled person in four questionnaire items. Specifically, these items were eye contact, vocal pauses, self disclosure, and vocalized questions. These specific items appear to have tapped verbal and nonverbal behaviors used most often as information about self. 
Perhaps eye contact was found to be statistically significant because it is often used by communicators to reflect interest in the other. According to Goffman (1963), ablebodied people will either engage in more eye contact, or less eye contact, depending on how they engage in or "ignore" the disability. The heightened awareness of the amount of eye contact reflects Goffman's thesis.

surprisingly, the majority of the questionnaire items' direction of mean differences failed to substantiate the second hypothesis. For the most part, there was less selfmonitoring of behavior in the disabled mode of conversation than the ablebodied mode. As discussed in Hypothesis I, this could be a function of several factors.

The author made the initial attempt to connect previous work on self-monitoring activity with transactions with disabled persons. In one study, hyper-self-monitoring activity had been found to cause a dysfunction in communication, creating a more formal, inhibited interaction (Lofland, 1976). Conversations with a disabled person were often found to be more formal and inhibited (Kleck, 1975). Davis (1961) and Goffman (1963) had found the ablebodied partner tended to carefully choose words which were thought to be less offensive to the stigmatized other; e.g., avoiding words like "walk." The implication seemed to be that the ablebodied person had heightened awareness of her own behavior to the point of extreme "self-consciousness" or 
dysfunction. This may not be the case. Perhaps self-monitoring is heightened in selective items, leading not to the dysfunction in competence, but to a sensitivity to the other. The inhibited, polite interactions perhaps reflect a separate variable outside of self-monitoring.

All subjects verbalized an interest in participating in this study, suggesting that their motivation was high. This heightened motivation could reflect a strong desire on the subject's part to help in the research, creating a more homogenous set of communicator behavior among the participants. Also, this may have heightened subjects' sensitivity to the partner and the situation.

As addressed in the first hypothesis, the selection of category limits for the adjective questionnaire items were subjective and somewhat arbitrary. A different limit selection may have changed the outcome in this study a great deal.

Hypothesis III

This hypothesis supported the connection between uncertainty reduction, attributional processes, and transactions with disabled persons. As predicted, mean differences in recall of information about partner were found to be less during TADs than TABs.

With a possible increase of anxiety felt by subjects during their conversations with a disabled partner (Davis, 
1961; Goffman, 1963; Kleck, Ono \& Hastoff, 1966), information was missed about the other. This increase of subject's anxiety would reduce attention to recall of the partner's behavior (Berger \& Calabrese, 1975). For whatever reasons, there occurred a significant loss, in certain specific aspects, of information about disabled partner's behavior versus ablebodied partner's behavior.

of the eighteen questionnaire items, ten were found to be in the predicted direction of mean difference, with less information recalled about the disabled partner. Of these ten items, two were found to be statistically significant (facial expression and voice type). Due to the increased awareness of the amount of eye contact by self during TADs (Hypothesis II), it is possible that what subjects were observing (facial expression) was secondary. For example, awareness of partner's facial expression was found to be significantly lower than with ablebodied partners. Of the nonverbal behaviors used to gain information about the other, facial expression is most often used (Ekman \& Friesen, 1969). It is uncertain why voice type was found to be statistically significantly lower in interactions with disabled people.

Although the majority of the mean differences in the questionnaire items supported the third hypothesis, direction of mean differences in eight of the eighteen items did not support this hypothesis. As addressed previously in 
this chapter, the reasons could be many. Since all subjects were selected from speech Communication courses, and confederates were graduate students in the same area, the topic choices were often focused on education and, specifically, speech communication. This may have produced perceptions of high homophily, which could have reduced the stress of the disabled interaction, thereby curtailing the predicted relationships among self-monitoring, uncertainty, uncertainty reduction, and ultimately, information about the partner.

Also addressed previously, individual questionnaire items may not reflect relevant specific behaviors used by each subject to reduce uncertainty. This is why analyses on individual recalled behaviors (items) were conducted. In addition, assessing abstract cognitive processes by inferring from pen and paper recall measures may be insufficient. That is, the domain of behaviors which are truly representative of the content of those constructs may not have been "captured" by the particular combination of specific test items used here.

General Discussion and Suggestions for Future Research

Many aspects not specifically tapped in this study reflected findings in previous work. For example, subjects tended to use relatively less gestural activity when interacting with the disabled partner. While gestural activity 
is often reciprocated, fewer hand gestures and torso movements were used by subject, regardless of the amount used by the disabled partner.

subjects often believed they were self-disclosing in a personal manner, when coders found it to be impersonal. For example, one subject talked about her partner's disability, which was believed to be personal disclosure, but the discussion was on an abstract and theoretical level. Her own feelings about that specific person, her disability, or disabilities in general were not discussed.

The experimental room design did not allow subjects to move either farther away or closer to their partner, due to the scope of the video camera. Many subjects turned their bodies away or slumped back in their chair in an attempt to gain distance from the disabled partner. This behavior was less apparent during the conversations with an ablebodied partner. None of the subjects "leaned-in" to the confederate who was believed to be disabled. This supports previous work on TADs which finds ablebodied persons tend to place a greater distance between themselves and a disabled partner.

A general observation by coders was that when interacting with the disabled partner, subjects tended to ask more questions and interrupt more. This reflects Goffman's (1963) theory on the stigmatization of a disabled person. stigmatized others are often attributed lower status, thus 
there is more freedom to ask questions and to interrupt them.

overall, while this study did not substantiate the first and second hypotheses, it did support the third hypothesis, which stated there would be less information recall during TADs and thereby supported previous research on behavioral differences of ablebodied persons during these transactions. This effort attempted to connect several different theories for the first time with respect to communication with disabled people. A heuristic model was constructed to investigate these possible connections. Uncertainty reduction, attributional processes, and self-monitoring activity were all related to transactions with disabled people.

From this study and previous research reflected in this study, there are several suggestions to be be made for future research. First, further research in self-monitoring activity and its relationship with communication competence should be conducted. Second, in this investigation a number of specific partner behaviors and the total vocal/ verbal dimension were recalled more in ablebodied than in disabled encounters and to statistically significant degree, as predicted. These findings need to be subject to the test of replication, as they furnish specific direction for future theory and research. 
A third approach would be to probe each variable suggested in the heuristic model (see Figure 1). The model should be modified and the strength and direction of each variable should be assessed, with possible exploration of variables not included in the old model. Ideally, a path analysis should be performed on the revised model. Finally, "attitude-toward-disabled-individuals" could be probed for its utility in a revised model. Although several outcomes did not conform to expected results, the present study did add some concrete information and several strategies for future research to the growing literature on communication with people who are disabled. 


\section{BIBLIOGRAPHY}

Berger, C. (1973). The acquaintance process revisited: explorations in initial interactions. Paper presented at Convention of Speech Communication Association, New York, November.

- (1975). Proactive and retroactive attribution processes in interpersonal communication. Human Comrunication Research, 2, 33-50.

Berger, C. \& Calabrese, R. (1975). Some explorations in initial interaction and beyond: toward a developmental theory on interpersonal communication. Human Communication Research, 99-]22.

Berger, C. \& Douglas, C. (1982). Thought and talk:

"excuse me, but have I been talking to myself?" In F.E.X. Dance (ed:) Human Communication Theory: Comparative Essays, New York, N.Y.: Harper and Row $(42-60)$.

Berger, C. \& Lamar, W. (1976). When beauty is only skin deep: the effects of physical attractiveness, sex, and time of initial interaction. Paper presented at the annual convention of the International communication Association, New Orleans.

Burgoon, J. \& Saine, T. (1978). The unspoken dialogue: an introduction to nonverbal communication. Boston, Mass: Houghton Mifflin.

Byrde, D. (1961). Interpersonal attraction and attitude similarity. Journal of Abnormal and Social

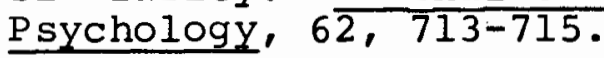

Byrde, D. \& Wong, T. (1962). Racial prejudice, interpersonal attraction and assumed dissimilarity of attitudes. Journal of Abnormal and Social Psychology, 65, 246-253.

- (1980). Communications: A Key to Understanding. President's Committee on Employment of the Handicapped, Annual Report, February.

Clatterbuck, G. (1979). Attributional confidence and uncertainty in initial interaction. Human Communication Research, 5, 2, winter. 
Dahnke, G. (1983). Communication between handicapped and nonhandicapped persons: toward a deductive theory. Communication Yearbook, 6(3), 92-135.

Davis, F. (1961). Deviance disavowal: the management of strained interaction by the visibly handicapped. Social Problems, 9, 120-132.

Ekrnan, P. \& Friesen; W. (1969). Nonverbal leakage and clues to deception. Psychiatry, 32, 88-106.

Festinger, L. (1961). A theory of comparison processes. Human Relations, 7, 117-140.

Gardner, J. \& Radel, M. (1978). Portrait of the disabled in the media. Journal of Community Psychology, $6(3)$, 269-274.

Gleidrnan, J. (1979). The wheelchair rebellion. Psychology Today, Aud., 59-66.

Goffman, E. (1963). Stigma: Notes on the Management of a Spoiled Identity, Englewood Cliffs, N.J.: PrenticeHall.

- (1967). Interaction ritual: essays on face-to-face behavior, New York, N.Y.: Paritheon.

Heider, F. (1958). The Psychology of Interpersonal Relations, New York, N.Y.: Wiley.

- (1980). How to Communicate to and about people who happen to be disabled. President's Committee on the Employment of the Handicapped, Seminar report, Washington, D.C., April.

Hurt, H. T, \& Cook, J. (1979). The impact of communicatively handicapped students on high school teacher's expectations in regular classrooms. Communication Quarterly, 27.

Jones, E. \& Davis, K. (1965). From acts to disposition: the attribution process in person perception. In Berkowitz (ed.), Advances in Experimental Social Psychology, (Vol 2.), New York, N.Y.: Academic Press, 219-266.

Jones, E, and others. (1971). Attribution: Perceiving the Causes of Behavior, General Learning Press. 
Kanouse, D., and others. (1971). Attribution: Perceiving the Causes of Behavior, General Learning Press.

Kelley, H., and others. (1971). Attribution: Perceiving the Causes of Behavior, General Learning Press.

Kleck, R. (1966). Emotional arousal in interactions with stigmatized persons. Psychology Report, 1226.

- (1976). Physical stigma and task-oriented interactions. Human Relations, 22 .

- (1975). Reactions to the handicapped -- sweaty palms and saccharine words. Psychology Today, 9, (Nov.), 122-124.

Kleck, R., Ono, H. \& Hastorf, A. (1966). The elfects of physical deviance upon face-to-face interaction. Humán Relations, 19, 425-436.

Kleck, R. \& Richardson, S. (1974). Physical appearance cues and interpersonal attraction in children. Child Development, 45, 305-310.

Kleck, R. \& Rubenstein, C. (1974). Physical attractiveness, perceived attitude similarity, and attraction in opposite-sex encounter. Journal of Personality and Social Psychology.

Lange, J. (1973). Rhetorical sensitivity and understanding. aper presented at the meeting of the Western Speech Communication Association, Portland, Oregon.

Lange, J. \& Grove, T. (1981) Sociometric and autononic responses to three levels of self-disclosure in dyads: a research note. Western Journal of Speech Cormunication, 45 (Fall), 355-362.

Langer, E.J. (1978). Rethinking the role of thought in social interaction. In J.H. Harvey. W.J. Ickes, R. F. Kidd (eäs.) New Directions in Attribution Research, Hillsdale, N.J.: Eribaum, Vo1. 2, 35-58.

Lerner, M.J. \& Becker, S.W. (1982) Interpersonal choice as a function of ascribed similarities and definition of the situation. Human Relations, 15, 27-34.

Lerner, M.J. \& Simmons, C.H. (1966). Observer's reaction to the "innocent victim": compassion or rejection? Journal of Personality and Social Psychology, 4, 203-210. 
Loftland, J. (1976). Doing social life: the qualitative study of human interaction in natural settings, New York, N.Y.: Wiley.

Marinelli, R. \& Orto, A. (1977). The psychological and social impact of physical disabilities, New York, N.Y.: Springer.

Mehrabian, A. (1971). Verbal and nonverbal interaction of strangers in a waiting situation. Journal of Experirental Research in Personality, 5, $\overline{127-138}$.

Heissenger, A. ,'Thoeson, R. \& Butler, A. (1967). Relationship of self concept to impact and obviousness of disability among male and female adolescents. Perceptual and Hotor Skills, 24 .

Newcomb, T.M. (1956). The prediction of interpersonal attraction. American Psychologist, 11, 575-586.

Nie, and others."(1975). Statistical Packages for the Social Sciences, 2nd ed., New York, N.Y.: McGrawHill.

Novack D. \& Lerner, M. (1968). Rejection as a consequence of perceived dissimilarity. Journal of Personality and Social Psychology, 9, 147-152.

- (1975). One in Eleven. President's Conmittee on the Employlient of the Handicapped, April.

Planalp, S. \& Honeycutt, J. (1984). When Uncertainty Increases in Relationships, Presented at meeting at the International Communication Association Convention, San Francisco.

- (1978). Presenting...Disabled People. President's Committee on the Employment of the Handicapped, Annual Meeting, February.

Richardson, S. (1971). Research report: handicap, appearance and stigma. Social Science and Medicine, Vol. 5 .

Richardson, S., and others. (1961). Cultural uniformity in reaction to physical disabilities. American Sociological Review, 26, 241-247.

Ruffner, R. (1982). Image and identity: marketing disabled adults in the media. Disabled USA, April, 5-7. 
Synder, M. (1974). Self-monitoring of expressive behavior. Journal of Personality and Social Psychology, 30, 526-537.

Spitzberg, B. \& Cupach, W. (1984). Interpersonal Communication Competence, Sage Publications.

Thompson, T.L. (1982). "You can't play marbles -- you have a wooden hand": communication with the handicapped. Communication Quarterly, 30(2), Spring, 108-115.

Van Riper, C. \& Emerson, I. (1984). Speech and Man, Englewood Cliffs, N.J.: Prentice-Hall.

Walster, E. (1966). Assignment of responsibility for an accident. Journal of Personality and Social Psychology, 3, 73-79.

Winter, M \& DeSimone, D. (1982). I'm a person, not a wheelchair! Problems of disabled adolescents. In Jones (ed.) Growing Up Disabled, ERIC: Council for Excpetional Children. 
APPENDIX A

INFORMED CONSENT

I, - hereby agree to serve as a subject in the research project entitled "Behaviors Between strangers in Initial Encounters" conducted by Doris Werkman.

I understand that the study involves meeting with two different strangers in separate encounters and that each interaction will be videotaped. I will then be asked to complete three short questionnaires pertaining to the two interactions.

I understand that this study will take approximately 30 minutes to complete.

It has been explained to me that the purpose of the study is to learn how strangers interact in initial encounters.

I may not receive any direct benefit from participation in this study, but my participation may help to increase knowledge which may benefit others in the future. Doris Werkman has offered to answer any questions I may have about the study. I have been assured that all information I give will be kept confidential and that the identity of all subjects will remain anonymous. 
I understand that I am free to withdraw from participation in this study at any time without jeopardizing my relationship with portland state university or the grade in my class.

I have read and understand the foregoing information. Date Signature 
PARTNER'S APPEARANCE AND NONVERBAL BEHAVIOR (PANB)

The following questions are to be answered as they pertain to your partner's appearance and behavior. Please check one.

1. HAIR COLOR: What was the color of your partner's hair?

_black dark brown $_{\text {Light brown }}$ dark blond ${ }^{\text {light blond }}$

2. GLASSES: Did your partner wear glasses?

Yes No

3. SHOES: What type of shoes did your partner wear? Sport shoes

_casual (clogs, loafers, leather tied, sandals) _Dress (low and high heels)

4. CLOTHING: What type of clothing did your partner wear?

_ Jeans __ _ _lacks skirt or dress

5. HAIR LENGTH: How long was your partner's hair? short (to ears)

Medium (to shoulders)

Long (below shoulders) 
6. BODY TYPE: What type of body build did your partner have?

Slight (underweight)

Heavy (overweight) Medium (just right)

For the remainder of this questionnaire, please compare this interaction to other interactions in which you have been involved.

7. EYE CONTACT: For the most part of this conversation, how often did your partner look at you? very little

A moderate amount

A great deal

8. POSTURE: For the most part of this conversation, what type of posture did your partner have?

_Llumped Erect _Leaning in"

9. GeSTURAL ACTIVITY: For the most part of this conversation, how much did your partner move her head, shoulders, arms and hands?

Very little

A moderate amount

A great deal 
10. FACIAL EXPRESSION: For the most part of this conversation, how much change in facial activity did your partner have (for example, frowning to smiling to surprise)?

Very little

A moderate amount

A great deal

11. List any nonverbal behaviors of your partner you may recall: 
APPENDIX C

PARTNER'S VOCAL AND VERBAL BEHAVIOR (PVVB)

The following ten questions are to be answered as they pertain to your partner's behavior. Please check one lexcept for question \#2).

1. TOPIC CHANGE: How many times did your partner initiate a new subject?

_-2 times _-3-5 times _over 5 times

2. TOPIC IDENTITY: Identify the subjects initiated by your partner.

3. SELF-DISCLOSURE: How much of your partner's comments were personal matters?

more personal than impersonal

more impersonal than personal equal amounts of personal/impersonal

4. AGREEMENT: How often did your partner orally express agreement with you?

_ $0-2$ times _ _ $3-5$ times over 5 times

5. INTERRUPTIONS: How often did your partner interrupt you?

-

3-5 times over 5 times 
6. QUESTIONS: How many questions did your partner ask you?

_-2 times _-3-5 times _ over 5 times

7. VOICE: For the most part of this conversation, what type of voice did your partner use? Quiet Medium Loud

8. VOCAL VARIETY: For the most part of this conversation, how much vocal animation did your partner have (for example, change in pitch, rate, volume, rhythm)? Very 1 ittle

A medium amount

A great deal

9. RATE OF SPEECH: For the most part of this conversation, what was your partner's rate of speech?

_L L Medium Fast

10. PAUSES: For the most part of this conversation, how many vocalized pauses did your partner use (for example, ums, ers, ya knows)?

_ $0-2$ times _ $3-5$ times over 5 times 
APPENDIX D

SELF BEHAVIOR (SB)

The following questions are to be answered as they pertain to your own behavior. Please check one (except for \#6).

1. EYE CONTACT: For the most part of this conversation, how often did you look at your partner? very little

A medium amount

A great deal

2. POSTURE: For the most part of this conversation, what type of posture did you have?

_Llumped ___ Erect "Leaning in"

3. GESTURAL ACTIVITY: For the most part of this conversation, how much did you move your head, shoulders, arms and hands?

very little

A medium amount

A great deal

4. FACIAL ACTIVITY: For the most part of this conversation, how much change in facial activity did you have (for example, frowning to smiling to surprise)? very little

A medium amount

A great deal 
5. TOPIC CHANGE: HOW many times did you initiate a new subject?

_-2 times _-3-5 times _over 5 times

6. TOPIC IDENTITY: Identify the subjects you initiated.

7. PAUSES: For the most part of this conversation, how many vocalized pauses did you use (for example, ums, ers, ya knows)?

_ $0-2$ times _ $3-5$ times _over 5 times

8. SELF-DISCLOSURE: HOw much of your comments were personal matters?

More personal than impersonal

More impersonal than personal

Equal amounts personal/impersonal

9. INTERRUPTIONS: How often did you interrupt your partner?

_-2 times _ _ $3-5$ times over 5 times

10. AGREEMENT: How often did you orally express agreement with your partner?

0-2 times

3-5 times over 5 times

11. QUESTIONS: How many questions did you ask your partner?

_-2 times _ $3-5$ times_over 5 times

12. List any nonverbal behaviors of yourself you may recall. 


\section{APPENDIX E}

CODING FORM

Interaction \# a b Mode $A B$ DS

subjects :

Eye Contact

Posture

Gestures

Expression

Topic Change

Pauses

Self Disclosure (P)

(I)

Interruptions

Agreement

Questions

Topic Identity

Comments :
Confederates:

Eye contact

Posture

Gestures

Expression

Topic Change

Pauses

Self Disclosure (P)

(I)

Inter ruptions

Agreement

Questions

Voice

Vocal variety

Rate

Topic Identity

Hair Color 
Confederates (continued):

Glasses

shoes

Clothing

Hair Length

Body Type

Comments: 


\section{APPENDIX F}

\section{DATA FORMS}

The following questions are to be answered as they pertain to your partner's appearance and behavior. Please check one.

1. HAIR COLOR: What was the color of your partner's hair?

_black

dark brown light brown

__ark blond light blond reddish

2. GLASSES: Did your partner wear glasses?

Yes No

3. SHOES: What type of shoes did your partner wear?

Sport shoes

_casual (clogs, loafers, leather tied, sandals)

Dress (low and high heels)

4. CLOTHING: What type of clothing did your partner wear?

_ Jeans Slacks __ Skirt or dress

5. HAIR LENGTH: How long was your partner's hair? Short (to ears) Medium (to shoulders) Long (below shoulders) 
6. BODY TYPE: What type of body build did your partner have?

Slight (underweight)

Heavy (overweight)

Medium (just right)

For the remainder of this questionnaire, please compare this interaction to other interactions in which you have been involved.

7. EYE CONTACT: For the most part of this conversation, how often did your partner look at you?

Lery little

A moderate amount

A great deal

8. POSTURE: For the most part of this conversation, what type of posture did your partner have?

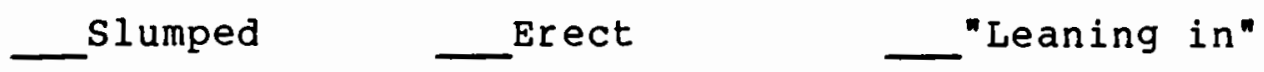

9. GESTURAL ACTIVITY: For the most part of this conversation, how much did your partner move her head, shoulders, arms and hands?

Very little

A moderate amount

A great deal 
10. FACIAL EXPRESSION: For the most part of this conversation, how much change in facial activity did your partner have (for example, frowning to smiling to surprise)?

Very little

A moderate amount

A great deal

11. List any nonverbal behaviors of your partner you may recall: 
The following ten questions are to be answered as they pertain to your partner's behavior. Please check one (except for question \#2).

1. TOPIC CHANGE: How many times did your partner initiate a new subject?

_-2 times _- $3-5$ times over 5 times

2. TOPIC IDENTITY: Identify the subjects initiated by your partner.

3. SELF-DISCLOSURE: How much of your partner's comments were personal matters? more personal than impersonal more impersonal than personal equal amounts of personal/impersonal

4. AGREEMENT: How often did your partner orally express agreement with you?

_-2 times _ _ $3-5$ times over 5 times

5. INTERRUPTIONS: How often did your partner interrupt you?

_ $0-2$ times _ $3-5$ times _over 5 times

6. QUESTIONS: How many questions did your partner ask you?

_-2 times _- $3-5$ times _over 5 times

7. VOICE: For the most part of this conversation, what type of voice did your partner use? Quiet Medium Loud 
8. VOCAL VARIETY: For the most part of this conversation, how much vocal animation did your partner have (for example, change in pitch, rate, volume, rhythm)? very little

A medium amount

A great deal

9. RATE OF SPEECH: For the most part of this conversation, what was your partner's rate of speech?

_Slow Medium Fast

10. PAUSES: For the most part of this conversation, how many vocalized pauses did your partner use (for example, ums, ers, ya knows)?

_ $0-2$ times _ $3-5$ times _ over 5 times 
The following questions are to be answered as they pertain to your own behavior. Please check one lexcept for \#6).

1. EYE CONTACT: For the most part of this conversation, how often did you look at your partner? Very little

A medium amount

A great deal

2. POSTURE: For the most part of this conversation, what type of posture did you have?

slumped

Erect

"Leaning in"

3. GESTURAL ACTIVITY: For the most part of this conversation, how much did you move your head, shoulders, arms and hands?

Very little

A medium amount

A great deal

4. FACIAL ACTIVITY: For the most part of this conversation, how much change in facial activity did you have (for example, frowning to smiling to surprise)? Very little

A medium amount

A great deal

5. TOPIC CHANGE: How many times did you initiate a new subject?

_ $^{0-2}$ times _- ${ }^{3-5}$ times $\quad$ over 5 times 
6. TOPIC IDENTITY: Identify the subjects you initiated.

7. PAUSES: For the most part of this conversation, how many vocalized pauses did you use (for example, ums, ers, ya knows)?

_0-2 times _- ${ }^{3-5}$ times over 5 times

8. SELF-DISCLOSURE: How much of your comments were personal matters?

More personal than impersonal

More impersonal than personal

Equal amounts personal/impersonal

9. INTERRUPTIONS: How often did you interrupt your partner?

_ $^{0-2}$ times ${ }^{3-5}$ times over 5 times

10. AGREEMENT: How often did you orally express agreement with your partner?

_ 0-2 times _-3-5 times _over 5 times

11. QUESTIONS: How many questions did you ask your partner?

_-2 times _-3-5 times _over 5 times

12. List any nonverbal behaviors of yourself you may recall. 